\title{
The Acoustic Analogy and Alternative Theories for Jet Noise Prediction
}

\author{
Philip J. Morris* \\ Department of Aerospace Engineering \\ The Pennsylvania State University \\ University Park, PA 16802, U.S.A. \\ and \\ F. Farassat ${ }^{\dagger}$ \\ Aeroacoustics Branch \\ NASA Langley Research Center \\ Hampton, VA 23681, U.S.A.
}

${ }^{*}$ Boeing/A. D. Welliver Professor, Associate Fellow, AIAA

†Senior Research Scientist, Associate Fellow, AIAA 


\begin{abstract}
This paper describes several methods for the prediction of jet noise. All but one of the noise prediction schemes are based on Lighthill's or Lilley's acoustic analogy while the other is the jet noise generation model recently proposed by Tam and Auriault. ${ }^{1}$ In all the approaches some assumptions must be made concerning the statistical properties of the turbulent sources. In each case the characteristic scales of the turbulence are obtained from a solution of the Reynolds-averaged Navier Stokes equation using a $k-\epsilon$ turbulence model. It is shown that, for the same level of empiricism, Tam and Auriault's model yields better agreement with experimental noise measurements than the acoustic analogy. It is then shown that this result is not because of some fundamental flaw in the acoustic analogy approach: but, is associated with the assumptions made in the approximation of the turbulent source statistics. If consistent assumptions are made, both the acoustic analogy and Tam and Auriault's model yield identical noise predictions. The paper concludes with a proposal for an acoustic analogy that provides a clearer identification of the equivalent source mechanisms and a discussion of noise prediction issues that remain to be resolved.
\end{abstract}

\title{
1 Introduction
}

The prediction of jet noise has been the object of continuous interest and study since the introduction of the jet engine for commercial use. The earliest theoretical formulation for aerodynamic noise was the work of Lighthill ${ }^{2,3}$. Lighthill's equation was the first example of an "acoustic analogy." The definition of an acoustic analogy, to be used in this paper, is any aerodynamic noise theory in which the equations of motion for a compressible fluid are rearranged in a way that seprates linear acoustic propagation effects. By definition, this rearrangement results in a set of equivalent sources that are assumed to be non-negligible in a limited region of space. In an apparent departure from formulations based on the acoustic analogy, Tam and Auriault ${ }^{1}$ have recently developed a jet noise prediction method in which 
the sound sources are modeled explicitly and the propagation of sound from these sources is described by solutions to the linearized Euler equations. These two aerodynamic noise theories appear to be very different. In this paper we reconcile these apparent differences and show that if consistent assumptions are made concerning the statistical properties of the turbulent noise sources, both approaches can yield identical noise predictions. It should be noted that this reconciliation has only been demonstrated at 90 degrees to the jet axis. The different approaches could lead to very different results at other angles.

In order to make predictions of the radiated noise it is necessary to describe the properties of the turbulence. Since a complete simulation of the jet turbulence and the noise it generates and radiates are too computationally expensive for high Reynolds number jet flows, noise predictions are often based on the solution of the Reynolds-averaged Navier Stokes (RANS) equations using a $k-\epsilon$ turbulence model. Such a solution provides an estimate of the amplitude of the turbulent velocity fluctuations as well as a local length scale. Since the $k-\epsilon$ solution only provides time-averaged properties, it is necessary to make assumptions about the statistical characteristics of the turbulence in order to predict the noise radiation that is an inherently unsteady phenomenon. In particular, the axial two-point cross correlation of the turbulent sources must be approximated. This correlation may be described in either a fixed frame of reference or a reference frame moving with the turbulence.

One of the earliest attempts to couple estimates of the statistical properties of the turbulence from a steady flow prediction with a noise model based on the acoustic analogy was the MGB approach developed by Mani, Gliebe and Balsa: see Balsa and Gliebe. ${ }^{4}$ More recent extensions have used RANS solutions for the flow field based on a $k-\epsilon$ turbulence model. This has been referred to as the MGBK method: see Khavaran et al. ${ }^{5}$ and Khavaran. ${ }^{6}$ In developing a solution to the acoustic analogy equations these methods assume a form for the two-point cross correlation function for the turbulent sources in a moving frame of reference. Conversely, Tam and Auriault, ${ }^{1}$ who also use RANS solutions for the flow field based on a $k-\epsilon$ turbulence model, describe the two-point cross correlation in a fixed reference frame. 
It is shown in this paper that, for the same RANS solution, jet noise predictions made with the Tam and Auriault model ${ }^{1}$ give much better agreement with experimental measurements at 90 degreees to the jet axis than methods based on the acosutic analogy. However, it will also be shown that this is not due to any inherent flaw in methods based on the acoustic analogy: but, is associated with the assumptions made concerning the statistical properties of the turbulent sources. Both approaches yield identical noise predictions at 90 degrees to the jet axis if consistent descriptions of the turbulent sources are chosen.

This paper is organized as follows. First a noise prediction formula based on Lighthill's acoustic analogy is developed. It is denoted here as Model I based on the acoustic analogy. The features of Tam and Auriault's noise prediction model ${ }^{1}$ are then described. Another model based on the solution to Lighthill's equation is then formulated using the solution procedure of Tam and Auriault. ${ }^{1}$ This is designated as Model II based on the acoustic analogy. The reasons for the differences between the noise predictions obtained with the different approaches are discussed and these differences are reconciled. Finally, an alternative form of acoustic analogy is proposed. It is argued that this form allows for the easier identification of the equivalent noise source mechanisms.

\section{Lighthill's Acoustic Analogy: Model I}

In this section we develop a prediction scheme based on the solution to Lighthill's equation. The details of the methodology do not follow those of the MGBK approach exactly. This is because the focus of the present paper is on noise radiation at 90 degrees to the jet axis, where mean flow/acoustic interaction effects are negligible. It is at this angle that the specification of the source and its assumed relationship to the $k-\epsilon$ solutions is best assessed. Also, it is intended to keep the assumptions made and the level of empiricism used as consistent as possible between the different schemes described in this paper. So, the possible importance of the effects of anisotropy of the turbulence on the radiated noise, as proposed by Khavaran, ${ }^{6}$ 
is not included. The analysis in this section follows that given by Goldstein ${ }^{7}$ and Lilley in Chapter 4 of Hubbard. ${ }^{8}$ It is repeated in sufficient detail here to emphasize any assumptions made and the differences with the alternative approaches presented in later sections of the paper.

Lighthill's equation ${ }^{2}$ may be written in Cartesian tensor form as,

$$
\frac{\partial^{2} \rho^{\prime}}{\partial t^{2}}-c_{o}^{2} \frac{\partial^{2} \rho^{\prime}}{\partial x_{i} \partial x_{i}}=\frac{\partial^{2} T_{i j}}{\partial x_{i} \partial x_{j}}
$$

where $T_{i j}$ is the Lighthill stress tensor given by

$$
T_{i j}=\rho u_{i} u_{j}+\delta_{i j}\left[\left(p-p_{o}\right)-c_{o}^{2}\left(\rho-\rho_{o}\right)\right]
$$

and primes denote perturbations about the basic state denoted by a subscript $o . c_{o}$ is a constant speed of sound that is sensibly taken to be the speed of sound in the uniform medium surrounding the source region. $u_{i}$ is the instantaneous velocity vector. Viscous terms have been neglected in the Lighthill stress tensor. In the subsequent analysis it is assumed that the departures from isentropic behavior are everywhere small and that the flow is at relatively low Mach number. Since we are concentrating on noise radiation at $90^{\circ}$ to the jet axis it is also assumed that the primary contributions to the Lighthill stress tensor involve products of velocity fluctuations. The terms that are linear in the fluctuations on the right hand side of Eqn. (1) should be regarded as terms associated with the propagation of the sound and be placed on the left hand side of the equation: see Lilley. ${ }^{9}$ These effects are negligible at $90^{\circ}$ to the jet axis so this assumption is reasonable here. So we approximate the Lighthill stress tensor by

$$
T_{i j}=\rho_{s} u_{i}^{\prime} u_{j}^{\prime}
$$

where $\rho_{s}$ is the mean density in the source region. In the far field the density fluctuation is 
readily shown to be given by

$$
\rho^{\prime}(\mathbf{x}, t)=\frac{1}{4 \pi c_{o}^{4}} \frac{1}{x} \iiint_{V(\mathbf{y})} \frac{\partial^{2}}{\partial t^{2}} T_{x x}\left(\mathbf{y}, t-\frac{|\mathbf{x}-\mathbf{y}|}{c_{o}}\right) d \mathbf{y}
$$

where $T_{x x}$ is the component of the Lighthill stress tensor in the direction of the far field observer and $x=|\mathbf{x}-\mathbf{y}| \approx|\mathbf{x}|$.

The far field spectral density for the intensity is related to the Fourier transform of the autocorrelation function of the far field pressure.

$$
S(\mathbf{x}, \omega)=\frac{1}{2 \pi} \int_{-\infty}^{\infty} \frac{\left\langle p^{\prime}(\mathbf{x}, t) p^{\prime}(\mathbf{x}, t+\tau)\right\rangle}{\rho_{o} c_{o}} e^{i \omega \tau} d \tau
$$

where \langle\rangle denotes an ensemble average. Since, in the far field, $p^{\prime}=c_{o}^{2} \rho^{\prime}$ we obtain

$$
S(\mathbf{x}, \omega)=\frac{1}{32 \pi^{3} \rho_{o} c_{o}^{5} x^{2}} \int_{-\infty}^{\infty} \int_{V\left(\mathbf{y}_{1}\right)} \int_{V\left(\mathbf{y}_{2}\right)}\left\langle\frac{\partial^{2} T_{x x}}{\partial t^{2}}\left(\mathbf{y}_{1}, t_{1}\right) \frac{\partial^{2} T_{x x}}{\partial t^{2}}\left(\mathbf{y}_{2}, t_{2}\right)\right\rangle e^{i \omega \tau} d \mathbf{y}_{1} d \mathbf{y}_{2} d \tau
$$

where

$$
\begin{aligned}
& t_{1}=t-\frac{\left|\mathbf{x}-\mathbf{y}_{1}\right|}{c_{o}} \\
& t_{2}=t+\tau-\frac{\left|\mathbf{x}-\mathbf{y}_{2}\right|}{c_{o}}
\end{aligned}
$$

If the turbulent statistics are assumed to be stationary and the usual far field approximation is made we obtain.

$$
S(\mathbf{x}, \omega)=\frac{1}{32 \pi^{3} \rho_{o} c_{o}^{5} x^{2}} \int_{-\infty}^{\infty} \int_{V\left(\mathbf{y}_{1}\right)} \int_{V\left(\mathbf{y}_{2}\right)} \frac{\partial^{4}}{\partial \tau^{4}}\left\langle T_{x x}\left(\mathbf{y}_{1}, t\right) T_{x x}\left(\mathbf{y}_{2}, \tau_{o}\right)\right\rangle e^{i \omega \tau} d \mathbf{y}_{1} d \mathbf{y}_{2} d \tau
$$


where

$$
\tau_{o}=t+\tau+\frac{\mathbf{x}}{x} \cdot \frac{\left(\mathbf{y}_{2}-\mathbf{y}_{1}\right)}{c_{o}}
$$

The two-point cross correlation function of the Lighthill stress tensor in a fixed reference frame may be denoted by,

$$
R_{f}\left(\mathbf{y}_{1}, \boldsymbol{\eta}, \tau\right)=\left\langle T_{x x}\left(\mathbf{y}_{1}, t\right) T_{x x}\left(\mathbf{y}_{2}, t+\tau\right)\right\rangle
$$

where $\boldsymbol{\eta}=\mathbf{y}_{2}-\mathbf{y}_{1}$, then,

$$
S(\mathbf{x}, \omega)=\frac{\omega^{4}}{32 \pi^{3} \rho_{o} c_{o}^{5} x^{2}} \int_{-\infty}^{\infty} \int_{V\left(\mathbf{y}_{1}\right)} \int_{V(\boldsymbol{\eta})} R_{f}\left(\mathbf{y}_{1}, \boldsymbol{\eta}, \tau\right) \exp \left(i \omega\left[\tau-\frac{\mathbf{x}}{x} \cdot \frac{\boldsymbol{\eta}}{c_{o}}\right]\right) d \mathbf{y}_{1} d \boldsymbol{\eta} d \tau
$$

As noted by Lighthill ${ }^{2,3}$ and others, it is best to include as many properties of the source as possible prior to any modeling of the turbulent sources. To include the effects of source convection the statistical properties of the sources may be described in a moving frame of reference. This also has the advantage that it is the temporal variation in this frame that controls the noise radiation. In a fixed reference frame, the temporal variation is dominated by convection effects. For example, as noted by Goldstein, ${ }^{7}$ a frozen pattern of turbulence convecting subsonically would radiate no noise. However, its local time variation would depend on the convection velocity and the turbulent length scales and would not be zero. Let

$$
\xi=\eta-\mathbf{i} c_{o} M_{c} \tau
$$

where, $\mathbf{i}$ is a unit vector in the direction of the mean flow and $M_{c}$ is the convection Mach 
number of the turbulent eddies. This gives

$$
\begin{aligned}
S(\mathbf{x}, \omega)= & \frac{\omega^{4}}{32 \pi^{3} \rho_{o} c_{o}^{5} x^{2}} \int_{-\infty}^{\infty} \int_{V\left(\mathbf{y}_{1}\right)} \int_{V(\xi)} \exp \left\{i \omega\left[\left(1-M_{c} \cos \theta\right) \tau-\frac{\mathbf{x}}{x} \cdot \frac{\boldsymbol{\xi}}{c_{o}}\right]\right\} \times \\
& R_{m}\left(\mathbf{y}_{1}, \boldsymbol{\xi}, \tau\right) d \boldsymbol{\xi} d \mathbf{y}_{1} d \tau
\end{aligned}
$$

where $R_{m}$ denotes the two-point cross correlation function of the Lighthill stress tensor in the moving reference frame. Also,

$$
\cos \theta=x_{1} / x
$$

The wavenumber/frequency spectrum of the turbulent sources is given by

$$
H\left(\mathbf{y}_{1}, \boldsymbol{\alpha}, \omega\right)=\frac{1}{(2 \pi)^{4}} \int_{V(\boldsymbol{\xi})} \int_{-\infty}^{\infty} e^{i(\omega \tau-\alpha \cdot \xi)} R_{m}\left(\mathbf{y}_{1}, \boldsymbol{\xi}, \tau\right) d \boldsymbol{\xi} d \tau
$$

where $\boldsymbol{\alpha}$ is a wavenumber vector. This describes the spatial and temporal periodicity of the source. Then,

$$
S(\mathbf{x}, \omega)=\frac{\pi \omega^{4}}{2 \rho_{o} c_{o}^{5}} \frac{1}{x^{2}} \int_{V\left(\mathbf{y}_{1}\right)} H\left[\mathbf{y}_{1}, \frac{\omega \mathbf{x}}{x c_{o}}, \omega\left(1-M_{c} \cos \theta\right)\right] d \mathbf{y}_{1}
$$

This shows that the far field noise depends on the components of the source wavenumber/frequency spectrum with a wavenumber that gives a sonic velocity in the direction of a far field observer and at a Doppler shifted frequency.

To this point, other than the far field assumption, no approximations have been made. However, to proceed further, it is necessary to introduce a model for the two point cross correlation. It is usually assumed that, in the moving frame of reference, the correlation 
takes on a Gaussian form (see Ffowcs Williams ${ }^{10}$ and Lilley $^{8}$ ),

$$
R_{m}\left(\mathbf{y}_{1}, \boldsymbol{\xi}, \tau\right)=A^{2} \rho_{s}^{2} u_{s}^{4} \exp \left[-\frac{|\boldsymbol{\xi}|^{2}}{\ell_{s}^{2}}-\omega_{s}^{2} \tau^{2}\right]
$$

where, $\ell_{s}$ is a characteristic length scale, $\omega_{s}$ is a characteristic frequency in the moving frame, $u_{s}$ is a velocity scale that characterizes the turbulent velocity fluctuations, and $A$ determines the magnitude of the correlation. Here, it assumed that the characteristic length scale is the same in all directions. This restriction could easily be relaxed. It should be noted that this is simply a model for the turbulent statistics in a moving reference frame. It is not an exact relationship. Then,

$$
\begin{aligned}
& H\left\{\mathbf{y}_{1}, \frac{\omega \mathbf{x}}{x c_{o}}, \omega\left(1-M_{c} \cos \theta\right)\right\} \\
= & \frac{A^{2}}{(2 \pi)^{4}} \rho_{s}^{2} u_{s}^{4} \frac{\pi^{2} \ell_{s}^{3}}{\omega_{s}} \exp \left\{-\frac{\omega^{2}\left(1-M_{c} \cos \theta\right)^{2}}{4 \omega_{s}^{2}}\right\} \exp \left\{-\frac{\left(\alpha \ell_{s}\right)^{2}}{4}\right\}
\end{aligned}
$$

where, $\alpha$ is the magnitude of the wavenumber vector. Now,

$$
\alpha \ell_{s}=\frac{\omega_{s} \ell_{s}}{c_{o}} \sim \frac{u_{s}}{c_{o}} \sim m
$$

where $m$ is a characteristic Mach number for the turbulence and provides a measure of the compactness of the source region. For compact sources, $m<<1$, so that at 90 degrees to the jet axis we obtain,

$$
S(\mathbf{x}, \omega)=\frac{A^{2}}{32 \pi \rho_{o} c_{o}^{5} x^{2}} \int_{V} \rho_{s}^{2} u_{s}^{4} \ell_{s}^{3} \omega_{s}^{3}\left(\frac{\omega}{\omega_{s}}\right)^{4} \exp \left\{-\frac{\omega^{2}}{4 \omega_{s}^{2}}\right\} d \mathbf{y}_{1}
$$

If a RANS $k-\varepsilon$ solution is available it is possible to determine the contribution of each elemental volume in the numerical grid to the radiated noise spectrum. Here, $k$ and $\epsilon$ are the turbulent kinetic energy and visous dissipation rate per unit mass respectively. From 
equation (21) this contribution is given by

$$
d S(\mathbf{x}, \omega)=\frac{A^{2}}{32 \pi \rho_{o} c_{o}^{5} x^{2}}\left\{\rho_{s}^{2} u_{s}^{4} \ell_{s}^{3} \omega_{s}^{3}\left(\frac{\omega}{\omega_{s}}\right)^{4} \exp \left(-\frac{\omega^{2}}{4 \omega_{s}^{2}}\right)\right\} d V
$$

The length and time scales may be obtained from the $k-\varepsilon$ solution. We assume that

$$
\omega_{s}=2 \pi / \tau_{s}, \quad \tau_{s}=c_{\tau}(k / \epsilon), \text { and } \quad \ell_{s}=c_{\ell}\left(k^{3 / 2} / \epsilon\right)
$$

The $k-\epsilon$ solutions indicate that, along the location of maximum shear, both $\tau_{s}$ and $\ell_{s}$ vary nearly linearly with axial distance. Then, with $u_{s}=\sqrt{2 k / 3}$,

$$
d S(\mathbf{x}, \omega)=\frac{A^{2} c_{\ell}^{3}}{c_{\tau}^{3}} \frac{\pi^{2}}{9 \rho_{o} c_{o}^{5} x^{2}}\left\{\rho_{s}^{2} k^{7 / 2}\left(\frac{\omega}{\omega_{s}}\right)^{4} \exp \left(-\frac{\omega^{2}}{4 \omega_{s}^{2}}\right)\right\} d V
$$

In this form there are only two combinations of constants that may be determined by comparison with experiments. The factor

$$
\left(\frac{\omega}{\omega_{s}}\right)^{4} \exp \left(-\frac{\omega^{2}}{4 \omega_{s}^{2}}\right)
$$

describes how each volume element contributes to a range of frequencies about the local characteristic frequency $\omega_{s}$.

Figure (1) shows a comparison of the predicted radiated noise with experimental data by Tanna et al. ${ }^{11}$ The one-third octave experimental data have been converted to spectral density assuming a smooth spectrum. The jet is operating at $M_{j}=0.911$ and $T_{j} / T_{o}=0.975$. The jet diameter is $0.0508 \mathrm{~m}$ and the observer location is at $90^{\circ}$ to the jet axis at a distance of 72 jet diameters. The $k-\epsilon$ solution has been obtained using the code developed by Thies and Tam. ${ }^{12}$ The grid is described by Thies and Tam. ${ }^{12}$ It grows in physical size in a stepwise manner as the solution is marched in the axial direction. For the present calculations the solutions are saved at every quarter of a jet diameter downstream. The volume of the 
elements is determined by the radial grid spacing, with a constant axial spacing and an axisymmetric assumption. The coefficients used in the noise predition are $c_{\tau}=1.43$ and $A^{2} c_{\ell}^{3}=1.93 \times 10^{-2}$. The predicted spectrum has been matched to the measured spectrum at the peak frequency. The predicted frequency variation of the spectrum increases as $f^{2}$ at low frequency and decreases as $f^{-2}$ at high frequencies. The much faster decay at very high frequencies is due to the lack of resolution in the flow prediction near the nozzle exit. It is clear that the behavior of the experiments is quite different. The experimental variation of the spectral density increases approximately with $f$ at low frequency and decreases as $f^{-1}$ at high frequencies. Figure (2) shows how each axial slice of length $D_{j}$ of the jet contributes to the total spectrum. The spectral shape of the contribution of each axial slice is determined by Eqn. (25). The slices near the jet exit provide the high frequency contributions to the spectrum and the peak frequencies of the spectra decrease monotonically in the axial drection. It is clear that this spectral distribution is unable to provide sufficiently high levels away from the peak, particularly at low frequencies.

\section{Tam and Auriault's Method}

In a recent development, Tam and Auriault ${ }^{1}$ developed a model for noise radiation from "small-scale" turbulence. As the name suggests, this model, they argued, accounts for the noise radiation by the small-scale components of the turbulence. In an earlier paper, Tam et al. ${ }^{13}$ had shown how experimental noise data for a wide range of jet operating conditions could be correlated extremely well with two shapes of spectral density function. One, with a well-defined peak and relatively rapid decay at high and low frequencies, was associated by Tam et al..$^{13}$ with noise generated by the large-scale structures in the turbulence. This spectrum matched the experimental noise data at small angles to the jet axis (including the peak noise direction). The second spectrum shape was much broader, similar to the measured spectrum shown in Fig. (1). This they associated with noise from the small scale turbulence 
and it matched the measured spectrum at larger angles to the jet axis. Tam and Auriault ${ }^{1}$ argue that their noise prediction model provides a description of the latter mechanism. It should be noted that there is no direct evidence that these two separate mechanisms are actually responsible for the total noise radiation: but, the experimental correlations and the success of the model can not be overlooked.

Before providing some of the details of Tam and Auriault's analysis, it is useful to summarize the differences between the model of the previous section based on the acoustic analogy and Tam and Auriault's model as well as the differences in the development of a prediction formula for the far field noise. Firstly, the Lighthill equation is based on the full equations of motion whereas Tam and Auriault's model contains a heuristic argument to describe the noise source and uses the linearized Euler equations to describe the propagation of sound generated by the model sources. In the model based on the acoustic analogy it is assumed that the source is compact and the two-point cross correlation function for the source is modeled in a moving reference frame. The Green's function in the model based on the acoustic analogy is simply the free space Green's function for the wave equation. In Tam and Auriault's method ${ }^{1}$ there is no explicit assumption concerning the compactness of the source, (though compactness is implied at one point), the two-point cross correlation function is formed in a fixed frame of reference, and the Green's function is obtained from the adjoint solution of the linearized Euler equations. A final difference between the two formulations is that the far field noise depends on a model for the cross correlation of the Lighthill stress tensor in the model based on the acoustic analogy and on the cross correlation of the convective derivative of the source term in Tam and Auriault's model. The consequence of this difference is examined in a later section of this paper.

In Tam and Auriault's model ${ }^{1}$ it is proposed that the small scale turbulence generates a local pressure fluctuation that is proportional to the local turbulent kinetic energy per unit 
volume. Tam and Auriault note that, in the kinetic theory of gases, the pressure is given by

$$
p=\frac{1}{3} m n\langle\mathbf{v} \cdot \mathbf{v}\rangle=\frac{1}{3} \rho\left\langle v^{2}\right\rangle
$$

where $\mathbf{v}$ is the random molecular velocity, $\rho$ is the density of the gas, and \langle\rangle denotes an ensemble average. Invoking a direct analogy, the small scale turbulence is considered as small blobs of fluid that interact in each element of the turbulent flow. They argue that the resulting pressure is related to the kinetic energy of the fine-scale turbulence. That is,

$$
p_{\text {turb }}=q_{s}=\frac{1}{3} \rho\left\langle v^{2}\right\rangle=\frac{2}{3} \rho k_{s}
$$

where $k_{s}$ is the kinetic energy of the small-scale turbulence per unit mass. It is argued that once sound is generated by these local pressure fluctuations, the propagation of the noise may be described by the linearized Euler equations with a "source" term on the right hand related to the fluctuating pressure gradient generated by the fine-scale turbulence. That is,

$$
\bar{\rho}\left[\frac{\partial u_{i}^{\prime}}{\partial t}+\bar{u}_{j} \frac{\partial u_{i}^{\prime}}{\partial x_{j}}+u_{j}^{\prime} \frac{\partial \bar{u}_{i}}{\partial x_{j}}\right]+\frac{\partial p^{\prime}}{\partial x_{j}}=-\frac{\partial q_{s}}{\partial x_{i}}
$$

where, an overbar denotes a mean quantity and $u_{i}^{\prime}$ and $p^{\prime}$ denote acoustic field variables. The sound waves are also assumed to satisfy the linearized equations of energy and continuity and the equation of state for a perfect gas. That is,

$$
\frac{\partial p}{\partial t}+\bar{u}_{j} \frac{\partial p}{\partial x_{j}}+\gamma \bar{p} \frac{\partial u_{i}}{\partial x_{i}}=0
$$

Tam and Auriault ${ }^{1}$ then write the component equations in cylindrical polar coordinates $(r, \phi, x)$ and assume that the mean flow is parallel.

Rather than converting the operators on the left hand sides of Eqns. (28) to (29) into the Lilley equation, ${ }^{9}$ Tam and Auriault ${ }^{1}$ make use of the adjoint equation. This is a very elegant 
approach to describe the mean flow acoustic interaction effects. A complete development for three-dimensional mean flows is given by Tam and Auriault. ${ }^{14}$ Tam and Auriault ${ }^{1}$ show that the periodic Green's function for the linearized Euler equations is related to the solution of the adjoint Euler equations by,

$$
\begin{aligned}
& \hat{p}_{1}\left(\mathbf{x}_{o}, \mathbf{x}_{1}, \omega\right)=u_{a}\left(\mathbf{x}_{1}, \mathbf{x}_{o}, \omega\right) \\
& \hat{p}_{2}\left(\mathbf{x}_{o}, \mathbf{x}_{1}, \omega\right)=v_{a}\left(\mathbf{x}_{1}, \mathbf{x}_{o}, \omega\right) \\
& \hat{p}_{3}\left(\mathbf{x}_{o}, \mathbf{x}_{1}, \omega\right)=w_{a}\left(\mathbf{x}_{1}, \mathbf{x}_{o}, \omega\right)
\end{aligned}
$$

$\hat{p}_{n}$ for $n=1,2,3$ are the Green's functions for sources in the $x, r$, and $\phi$ components of the linearized momentum equations respectively and. $u_{a}, v_{a}$, and $w_{a}$ are the solutions to the adjoint linearized momentum equations. Note the reciprocal dependence on $\mathbf{x}_{o}$ and $\mathbf{x}_{1}$. The adjoint equations may be written,

$$
\begin{aligned}
\bar{\rho}\left\{i \omega u_{a}+\bar{u} \frac{\partial u_{a}}{\partial x}\right\}+\gamma \bar{p} \frac{\partial p_{a}}{\partial x} & =0 \\
\bar{\rho}\left\{i \omega v_{a}+\bar{u} \frac{\partial v_{a}}{\partial x}-\frac{d \bar{u}}{d r} u_{a}\right\}+\frac{\gamma \bar{p}}{r} \frac{\partial\left(p_{a} r\right)}{\partial r} & =0 \\
\bar{\rho}\left\{i \omega w_{a}+\bar{u} \frac{\partial w_{a}}{\partial x}\right\}+\frac{\gamma \bar{p}}{r} \frac{\partial p_{a}}{\partial \phi} & =0 \\
\left(i \omega p_{a}+\bar{u} \frac{\partial p_{a}}{\partial x}\right)+\left[\frac{1}{r} \frac{\partial\left(v_{a} r\right)}{\partial r}+\frac{1}{r} \frac{\partial w_{a}}{\partial \phi}+\frac{\partial u_{a}}{\partial x}\right] & =-\frac{1}{2 \pi} \delta\left(\mathbf{x}-\mathbf{x}_{o}\right)
\end{aligned}
$$

With the Green's functions known, the general solution is given by the convolution of the Green's functions, or adjoint solutions, with the source terms. That is,

$$
\begin{aligned}
p^{\prime}(\mathbf{x}, t)= & -\iiint \iint\left\{\nabla_{1} \cdot\left[\mathbf{u}_{a}\left(\mathbf{x}_{1}, \mathbf{x}, \omega\right) q_{s}\left(\mathbf{x}_{1}, t_{1}\right)\right]\right. \\
& \left.-q_{s}\left(\mathbf{x}_{1}, t_{1}\right) \nabla_{1} \cdot\left[\mathbf{u}_{a}\left(\mathbf{x}_{1}, \mathbf{x}, \omega\right)\right]\right\} \times \exp \left[-i \omega\left(t-t_{1}\right)\right] d \omega d t_{1} d \mathbf{x}_{1}
\end{aligned}
$$


where

$$
\nabla_{1} \equiv\left(\frac{\partial}{\partial x_{1}}, \frac{\partial}{\partial r_{1}}, \frac{1}{r_{1}} \frac{\partial}{\partial \phi_{1}}\right)
$$

and

$$
\mathbf{u}_{a}\left(\mathbf{x}_{1}, \mathbf{x}_{o}, \omega\right)=\left[u_{a}, v_{a}, w_{a}\right]\left(\mathbf{x}_{1}, \mathbf{x}_{o}, \omega\right)
$$

However, the divergence of the adjoint velocity is known from the equation for the adjoint pressure $p_{a}$, given by Eqn. (34). Also, $q_{s}$ is zero at the source point for the adjoint solution, so that

$$
p^{\prime}(\mathbf{x}, t)=-\iiint \iint\left(i \omega p_{a}+\bar{u} \frac{\partial p_{a}}{\partial x_{1}}\right)\left(\mathbf{x}_{1}, \mathbf{x}, \omega\right) q_{s}\left(\mathbf{x}_{1}, t_{1}\right) \exp \left[-i \omega\left(t-t_{1}\right)\right] d \omega d t_{1} d \mathbf{x}_{1}
$$

or,

$$
p^{\prime}(x, t)=-\iiint \iint\left(\frac{\partial}{\partial t_{1}}+\bar{u} \frac{\partial}{\partial x_{1}}\right)\left\{p_{a} \exp \left[-i \omega\left(t-t_{1}\right)\right]\right\} q_{s}\left(\mathbf{x}_{1}, t_{1}\right) d \omega d t_{1} d \mathbf{x}_{1}
$$

It is important to note that, at this stage of the analysis, the convective derivative acts on the adjoint solution. However, integration by parts yields,

$$
p^{\prime}(\mathbf{x}, t)=\iiint \iint\left[p_{a}\left(\mathbf{x}_{1}, \mathbf{x}, \omega\right) \exp \left[-i \omega\left(t-t_{1}\right)\right]\right] \frac{D}{D t_{1}}\left\{q_{s}\left(\mathbf{x}_{1}, t_{1}\right)\right\} d \omega d t_{1} d \mathbf{x}_{1}
$$

where the convective derivative is given by

$$
\frac{D}{D t_{1}} \equiv\left(\frac{\partial}{\partial t_{1}}+\bar{u} \frac{\partial}{\partial x_{1}}\right)
$$

The spectral density for the intensity is then obtained by forming the autocorrelation for the pressure and taking it's Fourier transform, as given in Eqn. (5). The spectral density is 
then found to be given by

$$
\begin{aligned}
S(\mathbf{x}, \omega)= & \frac{1}{\rho_{o} c_{o}} \iiint \cdots \iiint p_{a}\left(\mathbf{x}_{1}, \mathbf{x}, \omega_{1}\right) p_{a}\left(\mathbf{x}_{2}, \mathbf{x}, \omega_{2}\right)\left\langle\frac{D}{D t_{1}}\left\{q_{s}\left(\mathbf{x}_{1}, t_{1}\right)\right\} \frac{D}{D t_{2}}\left\{q_{s}\left(\mathbf{x}_{2}, t_{2}\right)\right\}\right\rangle \\
& \times \exp \left[-i\left(\omega_{1}+\omega_{2}\right) t+i \omega_{1} t_{1}+i \omega_{2} t_{2}\right] \delta\left(\omega-\omega_{2}\right) d \omega_{1} d \omega_{2} d t_{1} d t_{2} d \mathbf{x}_{1} d \mathbf{x}_{2}
\end{aligned}
$$

Again, as in models based on the acoustic analogy, it is necessary to make some assumption about the correlation function for the source terms. Based on experimental measurements of the two-point cross correlation of the axial velocity fluctuations in a fixed reference frame, Tam and Auriault ${ }^{1}$ assume that

$$
\left\langle\frac{D}{D t_{1}}\left\{q_{s}\left(\mathbf{x}_{1}, t_{1}\right)\right\} \frac{D}{D t_{2}}\left\{q_{s}\left(\mathbf{x}_{2}, t_{2}\right)\right\}\right\rangle=\frac{\hat{q}_{s}^{2}}{c^{2} \tau_{s}^{2}} \exp \left\{-\frac{|\xi|}{\bar{u} \tau_{s}}-\frac{1}{\ell_{s}^{2}}\left[(\xi-\bar{u} \tau)^{2}+\eta^{2}+\zeta^{2}\right]\right\}
$$

where,

$$
\xi=x_{1}-x_{2}, \quad \eta=y_{1}-y_{2}, \quad \zeta=z_{1}-z_{2}, \quad \tau=t_{1}-t_{2}
$$

It should be noted again, as in Model I, that this is a major assumption and it is only supported indirectly by experimental measurements. In addition, the turbulent statistics are modeled in terms of the cross correlation of the convective derivative of the source. This is shown below to be a crucial choice in the formulation.

Following the sequence of integrations given by Tam and Auriault ${ }^{1}$ it is straightforward to show that

$$
\begin{aligned}
S(\mathbf{x}, \omega)= & \frac{2 \pi \sqrt{\pi}}{\rho_{o} c_{o}} \iiint \iiint p_{a}\left(\mathbf{x}_{1}, \mathbf{x},-\omega\right) p_{a}\left(\mathbf{x}_{2}, \mathbf{x}, \omega\right) \frac{\hat{q}_{s}^{2}}{c^{2} \tau_{s}^{2}} \frac{\ell_{s}}{\bar{u}} \\
& \times \exp \left\{-\frac{\omega^{2} \ell_{s}^{2}}{4 \bar{u}^{2}}\right\} \exp \left\{-\frac{|\xi|}{\bar{u} \tau_{s}}+i \omega_{1} \frac{\xi}{\bar{u}}-\frac{1}{\ell_{s}^{2}}\left[\eta^{2}+\zeta^{2}\right]\right\} d \mathbf{x}_{1} d \mathbf{x}_{2}
\end{aligned}
$$

Tam and Auriault ${ }^{1}$ argue that the difference between $p_{a}\left(\mathbf{x}_{1}, \mathbf{x},-\omega\right)$ and $p_{a}\left(\mathbf{x}_{2}, \mathbf{x}, \omega\right)$ is a 
simple phase factor such that

$$
p_{a}\left(\mathbf{x}_{1}, \mathbf{x},-\omega\right) \simeq p_{a}\left(\mathbf{x}_{2}, \mathbf{x}, \omega\right) \exp \left[-i \frac{\omega}{a_{\infty}} \cos \theta\left(x_{1}-x_{2}\right)\right]
$$

$\theta$ is the polar angle measured from the downstream jet axis. This implies that the sources have a limited spatial extent. That is, the length scale $\ell_{s}$ is small compared to the total extent of the source region. Thus, this model would not be appropriate for large scale or non compact sources. They also note that,

$$
p_{a}\left(\mathbf{x}_{2}, \mathbf{x},-\omega\right)=p_{a}^{*}\left(\mathbf{x}_{2}, \mathbf{x}, \omega\right)
$$

The integrations with respect to $\mathbf{x}_{1}$ may now be replaced by integrations with respect to the separation distance $\boldsymbol{\xi}=\mathbf{x}_{1}-\mathbf{x}_{2}$ The spectral density is then found to be given by,

$$
S(\mathbf{x}, \omega)=\frac{4 \pi^{2} \sqrt{\pi}}{\rho_{o} c_{o}} \iiint\left|p_{a}\left(\mathbf{x}_{2}, \mathbf{x}, \omega\right)\right|^{2} \frac{\hat{q}_{s}^{2}}{c^{2} \tau_{s}} \ell_{s}^{3} \frac{\exp \left\{-\omega^{2} \ell_{s}^{2} /\left(4 \bar{u}^{2}\right)\right\}}{\left[1+\omega^{2} \tau_{s}^{2}\left(1-\bar{u} \cos \theta / a_{\infty}\right)\right]} d \mathbf{x}_{2}
$$

In order to obtain a closed form result it is necessary to determine $\left|p_{a}\left(\mathbf{x}_{2}, \mathbf{x}, \omega\right)\right|^{2}$. Since at $\theta=\pi / 2$ the effects of the mean flow are negligible, the equations for the adjoint functions (31) to (34) may be reduced to a Helmholtz equation for the adjoint pressure,

$$
\nabla^{2} p_{a}+k^{2} p_{a}=\frac{i \omega}{2 \pi c_{o}^{2}} \delta\left(\mathbf{x}-\mathbf{x}_{2}\right)
$$

So that,

$$
\left|p_{a}\left(\mathbf{x}_{2}, \mathbf{x}, \omega\right)\right|^{2}=\frac{\omega^{2}}{64 \pi^{4} c_{o}^{4} x^{2}}
$$


Then, from equation (48), with $\theta=\pi / 2$, we obtain

$$
S(\mathbf{x}, \omega)=\frac{\sqrt{\pi}}{16 \pi^{2} \rho_{o} c_{o}^{5} x^{2}} \iiint \frac{\hat{q}_{s}^{2}}{c^{2} \tau_{s}} \ell_{s}^{3} \omega^{2} \frac{\exp \left\{-\omega^{2} \ell_{s}^{2} /\left(4 \bar{u}^{2}\right)\right\}}{\left(1+\omega^{2} \tau_{s}^{2}\right)} d \mathbf{x}_{2}
$$

With the scales given by Eqn. (23) and $\hat{q}_{s}^{2} / c^{2}=4 A^{2} \rho_{s}^{2} k^{2} / 9$, the contribution to the spectral density from an elemental volume of the turbulence is given by,

$$
d S(\mathbf{x}, \omega)=\frac{\sqrt{\pi}}{9 \rho_{o} c_{o}^{5} x^{2}} \frac{A^{2} c_{\ell}^{3}}{c_{\tau}^{3}} \rho_{s}^{2} k^{7 / 2} \frac{\left(\omega / \omega_{s}\right)^{2}}{\left(1+4 \pi^{2} \omega^{2} / \omega_{s}^{2}\right)} \exp \left\{-\frac{\pi^{2} c_{\ell}^{2}}{c_{\tau}^{2}} \frac{\omega^{2}}{\omega_{s}^{2}} \frac{k}{\bar{u}^{2}}\right\} d V
$$

This is very nearly the same result as given by the Lighthill Acoustic Analogy in equation (24) except for the frequency weighting function.

$$
\frac{\left(\omega / \omega_{s}\right)^{2}}{\left(1+4 \pi^{2} \omega^{2} / \omega_{s}^{2}\right)} \exp \left\{-\frac{\pi^{2} c_{\ell}^{2}}{c_{\tau}^{2}} \frac{\omega^{2}}{\omega_{s}^{2}} \frac{k}{\bar{u}^{2}}\right\}
$$

Figure 3 shows a comparison between predictions based on Tam and Auriault's model and experiments. The agreement between the predictions and measurement is excellent. This excellent agreement follows that shown by Tam and Auriault ${ }^{1}$ for a very wide set of jet operating conditions. The coefficients used in the present prediction based on Eqn. (52) are $c_{\tau}=0.308, c_{\ell}=0.130$, and $A=0.773$. These are similar to the coefficients determined by Tam and Auriault ${ }^{1}$. Also shown in Fig. 3 is a prediction of the spectral density based on the coefficients determined by Tam and Auriault ${ }^{1}$. It can be seen that their predicted spectral density falls more rapidly at higher frequencies than the present predicted spectrum or the measurements by Tanna et al. ${ }^{11}$ However, Tam and Auriault's predictions agree well with measurements in other facilities. This shows how the values of the coefficients are influenced by the choice of experimental data. However, for both sets of coefficients, the values are arguably more reasonable than the value of $c_{\tau}$ obtained in Model I that was greater than unity. 


\section{Lighthill's Acoustic Analogy: Model II}

In this section we will follow the same general approach used by Tam and Auriault: ${ }^{1}$ but, here we apply the method to Lighthill's acoustic analogy equation. The starting point is Eqn. (1). The Green's function is given by the solution of the equation

$$
\frac{\partial^{2} G}{\partial t^{2}}-c_{o}^{2} \frac{\partial^{2} G}{\partial x_{i} \partial x_{i}}=\delta\left(\mathbf{x}-\mathbf{x}_{1}\right) \delta\left(t-t_{1}\right)
$$

We seek a periodic Green's function such that

$$
G\left(\mathbf{x}, \mathbf{x}_{1}, t, t_{1}\right)=\int_{-\infty}^{\infty} \hat{G}\left(\mathbf{x}, \mathbf{x}_{1}, \omega\right) \exp \left[-i \omega\left(t-t_{1}\right)\right] d \omega
$$

Then, the Fourier transform of equation (54) gives

$$
\left(\nabla^{2}+k^{2}\right) \hat{G}\left(\mathbf{x}, \mathbf{x}_{1}, \omega\right)=\frac{\delta\left(\mathbf{x}-\mathbf{x}_{1}\right)}{2 \pi c_{o}^{2}}
$$

The adjoint function $G_{a}\left(\mathbf{x}, \mathbf{x}_{s}, \omega\right)$ now satisfies the equation,

$$
\left(\nabla^{2}+k^{2}\right) G_{a}\left(\mathbf{x}, \mathbf{x}_{s}, \omega\right)=-\frac{\delta\left(\mathbf{x}-\mathbf{x}_{s}\right)}{2 \pi c_{o}^{2}}
$$

and it is readily shown that,

$$
\hat{G}\left(\mathbf{x}_{s}, \mathbf{x}_{1}, \omega\right)=G_{a}\left(\mathbf{x}_{1}, \mathbf{x}_{s}, \omega\right)
$$

Thus the far field density may be written

$$
\rho^{\prime}(\mathbf{x}, t)=\iiint \iint G_{a}\left(\mathbf{x}_{1}, \mathbf{x}, \omega\right) \frac{\partial^{2} T_{i j}}{\partial x_{1 i} \partial x_{1 j}}\left(\mathbf{x}_{1}, \mathbf{t}_{1}\right) \exp \left[-i \omega\left(t-t_{1}\right)\right] d \omega d t_{1} d \mathbf{x}_{1}
$$


The subsequent analysis is simplified if the derivatives are transferred onto the adjoint function by integration by parts. That is,

$$
\rho^{\prime}(\mathbf{x}, t)=\iiint \iint \frac{\partial^{2} G_{a}}{\partial x_{1 i} \partial x_{1 j}}\left(\mathbf{x}_{1}, \mathbf{x}, \omega\right) T_{i j}\left(\mathbf{x}_{1}, \mathbf{t}_{1}\right) \exp \left[-i \omega\left(t-t_{1}\right)\right] d \omega d t_{1} d \mathbf{x}_{1}
$$

The spectral density for the intensity is defined by Eqn. (5) and is given by,

$$
\begin{aligned}
S(\mathbf{x}, \omega)= & \frac{c_{o}^{3}}{\rho_{o}} \iiint \cdots \iiint \frac{\partial^{2} G_{a}}{\partial x_{1 i} \partial x_{1 j}}\left(\mathbf{x}_{1}, \mathbf{x}, \omega_{1}\right) \frac{\partial^{2} G_{a}}{\partial x_{2 k} \partial x_{2 \ell}}\left(\mathbf{x}_{2}, \mathbf{x}, \omega_{2}\right)\left\langle T_{i j}\left(\mathbf{x}_{1}, \mathbf{t}_{1}\right) T_{k \ell}\left(\mathbf{x}_{2}, \mathbf{t}_{2}\right)\right\rangle \\
& \times \exp \left[-i \omega_{1}\left(t-t_{1}\right)-i \omega_{2}\left(t-t_{2}\right)\right] \delta\left(\omega-\omega_{2}\right) d \omega_{1} d \omega_{2} d t_{1} d t_{2} d \mathbf{x}_{1} d \mathbf{x}_{2}
\end{aligned}
$$

It is now assumed that the two point cross correlation of the Lighthill stress tensor in a fixed frame of reference is given by,

$$
\left\langle T_{i j}\left(\mathbf{x}_{1}, \mathbf{t}_{1}\right) T_{k \ell}\left(\mathbf{x}_{2}, \mathbf{t}_{2}\right)\right\rangle=A_{i j k \ell} \rho_{s}^{2} u_{s}^{4} \exp \left\{-\frac{|\xi|}{\bar{u} \tau_{s}}-\frac{1}{\ell_{s}^{2}}\left[(\xi-\bar{u} \tau)^{2}+\eta^{2}+\zeta^{2}\right]\right\}
$$

Then,

$$
\begin{aligned}
S(\mathbf{x}, \omega)= & \frac{c_{\boldsymbol{o}}^{3}}{\rho_{o}} \iiint \cdots \iiint \frac{\partial^{2} G_{a}}{\partial x_{1 i} \partial x_{1 j}}\left(\mathbf{x}_{1}, \mathbf{x}, \omega_{1}\right) \frac{\partial^{2} G_{a}}{\partial x_{2 k} \partial x_{2 \ell}}\left(\mathbf{x}_{2}, \mathbf{x}, \omega_{2}\right) \\
& \times A_{i j k \ell} \rho_{s}^{2} u_{s}^{4} \exp \left\{\left[-\frac{\left|x_{1}-x_{2}\right|}{\bar{u} \tau_{s}}-\frac{1}{\ell_{s}^{2}}\left(\left(x_{1}-x_{2}\right)-\bar{u}\left(t_{1}-t_{2}\right)\right)^{2}\right.\right. \\
& \left.\left.+\left(y_{1}-y_{2}\right)^{2}+\left(z_{1}-z_{2}\right)^{2}\right]\right\} \\
& \times \exp \left[-i\left(\omega_{1}+\omega_{2}\right) t+i \omega_{1} t_{1}+i \omega_{2} t_{2}\right] \delta\left(\omega-\omega_{2}\right) d \omega_{1} d \omega_{2} d t_{1} d t_{2} d \mathbf{x}_{1} d \mathbf{x}_{2}
\end{aligned}
$$

Again, following the sequence of integrations given by Tam and Auriault, ${ }^{1}$ we obtain,

$$
\begin{aligned}
S(\mathbf{x}, \omega)= & 2 \pi \sqrt{\pi} \frac{c_{o}^{3}}{\rho_{o}} \iiint \iiint \frac{\partial^{2} G_{a}}{\partial x_{1 i} \partial x_{1 j}}\left(\mathbf{x}_{1}, \mathbf{x},-\omega\right) \frac{\partial^{2} G_{a}}{\partial x_{2 k} \partial x_{2 \ell}}\left(\mathbf{x}_{2}, \mathbf{x}, \omega\right) A_{i j k \ell} \rho_{s}^{2} u_{s} \frac{\ell_{s}}{\bar{u}} \\
& \times \exp \left(-\frac{\omega^{2} \ell_{s}^{2}}{4 \bar{u}^{2}}\right) \exp \left\{-\frac{|\xi|}{\bar{u} \tau_{s}}-\frac{i \omega \xi}{\bar{u}}-\frac{1}{\ell_{s}^{2}}\left(\eta^{2}+\zeta^{2}\right)\right\} d \mathbf{x}_{1} d \mathbf{x}_{2}
\end{aligned}
$$


The solution to Eqn. (57) is

$$
G_{a}\left(\mathbf{x}_{1}, \mathbf{x},-\omega\right)=-\frac{1}{8 \pi^{2} c_{o}^{2}} \frac{\exp \left[i k\left|\mathbf{x}_{1}-\mathbf{x}\right|\right]}{\left|\mathbf{x}_{1}-\mathbf{x}\right|}
$$

It may be shown that as $\left|\mathbf{x}_{1}-\mathbf{x}\right| \rightarrow \infty$,

$$
\frac{\partial G_{a}}{\partial x_{1 i} \partial x_{1 j}}\left(\mathbf{x}_{1}, \mathbf{x},-\omega\right) \frac{\partial G_{a}}{\partial x_{2 k} \partial x_{2 \ell}}\left(\mathbf{x}_{2}, \mathbf{x}, \omega\right)=\frac{\omega^{4}}{64 \pi^{4} c_{o}^{8} x^{2}} \beta_{i} \beta_{j} \beta_{k} \beta_{\ell} \exp \left[i \omega\left(\mathbf{x}_{2}-\mathbf{x}_{1}\right) \cdot \frac{\mathbf{x}}{x c_{o}}\right]
$$

where $\beta_{i}=\left(x_{1 i}-x_{i}\right) / x$, etc. are direction cosines. Then, at $\theta=\pi / 2$ where, $\left(\mathbf{x}_{2}-\mathbf{x}_{1}\right) \cdot \mathbf{x} / x \approx$ 0 , the far field spectral density for the intensity may be written,

$$
\begin{aligned}
S(\mathbf{x}, \omega)= & \frac{\sqrt{\pi}}{32 \pi^{3}} \frac{\omega^{4}}{\rho_{o} c_{o}^{5} x^{2}} \iiint \iiint A_{2222} \rho_{s}^{2} u_{s}^{4} \frac{\ell_{s}}{\bar{u}} \\
& \times \exp \left(-\frac{\omega^{2} \ell_{s}^{2}}{4 \bar{u}^{2}}\right) \exp \left\{-\frac{|\xi|}{\bar{u} \tau_{s}}-\frac{i \omega \xi}{\bar{u}}-\frac{1}{\ell_{s}^{2}}\left(\eta^{2}+\zeta^{2}\right)\right\} d \boldsymbol{\xi} d \mathbf{x}_{2}
\end{aligned}
$$

With $A_{2222}=A^{2}$, and following the integrations with respect to $\boldsymbol{\xi}$, we obtain

$$
S(\mathbf{x}, \omega)=\frac{\sqrt{\pi}}{16 \pi^{2}} \frac{A^{2}}{\rho_{o} c_{o}^{5} x^{2}} \iiint \frac{\rho_{s}^{2} u_{s}^{4} \ell_{s}^{3}}{\tau_{s}^{3}} \frac{\left(\omega \tau_{s}\right)^{4}}{\left[1+\omega^{2} \tau_{s}^{2}\right]} \exp \left(-\frac{\omega^{2} \ell_{s}^{2}}{4 \bar{u}^{2}}\right) d \mathbf{x}_{2}
$$

Using the scales defined by Eqn. (23) and with $u_{s}=\sqrt{2 k / 3}$, the contribution to the spectral density from each volume element of the flow may be written,

$$
d S(\mathbf{x}, \omega)=\frac{\pi^{2} \sqrt{\pi}}{9 \rho_{o} c_{o}^{5} x^{2}} \frac{A^{2} c_{\ell}^{3}}{c_{\tau}^{3}} \rho_{s}^{2} k^{7 / 2} \frac{\left(\omega / \omega_{s}\right)^{4}}{\left(1+4 \pi^{2} \omega^{2} / \omega_{s}^{2}\right)} \exp \left(-\frac{\pi^{2} c_{\ell}^{2}}{c_{\tau}^{2}} \frac{\omega^{2}}{4 \omega_{s}^{2}} \frac{k}{\bar{u}^{2}}\right) d V
$$

This is essentially the same result obtained from Tam and Auriault's model ${ }^{1}$ except for the slight but significant change in the frequency weighting factor

$$
\frac{\left(\omega / \omega_{s}\right)^{4}}{\left(1+4 \pi^{2} \omega^{2} / \omega_{s}^{2}\right)} \exp \left(-\frac{\pi^{2} c_{\ell}^{2}}{c_{\tau}^{2}} \frac{\omega^{2}}{4 \omega_{s}^{2}} \frac{k}{\bar{u}^{2}}\right)
$$


Figure 4 shows a comparison of predictions made with all three models with experimental data. The coefficients used in the Model II prediction are, $c_{\tau}=1.0, c_{\ell}=0.78$, and $A=$ 0.8475. Clearly, the Model II prediction is an improvement over that made with the Model I version based on the acoustic analogy. Some of this is due to the additional freedom to specify three independent coefficients unlike the two available for Model I. However, the shape of the spectrum is still not as well predicted as that given by Tam and Auriault's model $^{1}$ for the same level of empiricism.

In the next section, we consider the final difference between the various models: the specification of the turbulent source statistics.

\section{Models for the Turbulent Source Statistics}

In the previous sections it was shown how a model based on the acoustic analogy failed to provide as good a set of noise predictions as the Tam and Auriault model ${ }^{1}$ even when the problems were formulated in the same manner. However, there remains one issue to be considered: how the statistics of the turbulent sources are described. Tam and Auriault model the source statistics with Eqn. (43) whereas the model of the last section used Eqn. (62). In the former case it is assumed that the cross correlation of the convective derivative of the source term follows the experimentally measured cross correlation of the axial velocity fluctuations in the jet. In the latter case, it is assumed that it is the cross correlation of the Lighthill stress tensor that follows the measured form. In this section the two prediction schemes are compared when consistent forms of the statistics of the turbulent source are used.

There are two ways to modify Tam and Auriault's model ${ }^{1}$ to make their assumptions consistent with Model II. The first is to retain the convective derivative operators on the adjoint solution in Eqn. (38). Once the form of the adjoint solution is obtained, the operators may be applied in a straightforward manner. However, in the general case, when the adjoint 
solution is obtained numerically, it is as convenient to still perform the integration by parts and use the form for the spectral density given by Eqn. (42). However, it is now assumed that the cross correlation of the source function may be written,

$$
\left\langle q_{s}\left(\mathbf{x}_{1}, t_{1}\right) q_{s}\left(\mathbf{x}_{2}, t_{2}\right)\right\rangle=\frac{\hat{q}_{s}^{2}}{c^{2}} \exp \left\{-\frac{|\xi|}{\bar{u} \tau_{s}}-\frac{1}{\ell_{s}^{2}}\left[(\xi-\bar{u} \tau)^{2}+\eta^{2}+\zeta^{2}\right]\right\}
$$

This is consistent with the assumption made in Eqn. (62) for Model II based on the acoustic analogy. The cross correlation required in Eqn. (42) is,

$$
\left\langle\frac{D q_{s}}{D t_{1}}\left(\mathbf{x}_{1}, t_{1}\right) \frac{D q_{s}}{D t_{2}}\left(\mathbf{x}_{2}, t_{2}\right)\right\rangle=\frac{D^{2}}{D t_{1} D t_{2}}\left\langle q_{s}\left(\mathbf{x}_{1}, t_{1}\right) q_{s}\left(\mathbf{x}_{2}, t_{2}\right)\right\rangle
$$

The derivatives may be written in terms of the time delay $\tau$ and the axial spatial separation distance $\xi$ such that,

$$
\frac{D^{2}}{D t_{1} D t_{2}} \equiv-\left(\frac{\partial}{\partial \tau}+\bar{u} \frac{\partial}{\partial \xi}\right)^{2}
$$

Now, if

$$
f=f(\xi-\bar{u} \tau) \quad \text { then } \quad\left(\frac{\partial}{\partial \tau}+\bar{u} \frac{\partial}{\partial \xi}\right) f=0
$$

Thus,

$$
\begin{aligned}
\left\langle\frac{D q_{s}}{D t_{1}}\left(\mathbf{x}_{1}, t_{1}\right) \frac{D q_{s}}{D t_{2}}\left(\mathbf{x}_{2}, t_{2}\right)\right\rangle \sim & -\frac{\hat{q}_{s}^{2}}{c^{2}} \bar{u}^{2} \exp \left\{-\frac{1}{\ell_{s}^{2}}\left[(\xi-\bar{u} \tau)^{2}+\eta^{2}+\zeta^{2}\right]\right\} \\
& \times \frac{\partial^{2}}{\partial \xi^{2}}\left[\exp \left(-\frac{|\xi|}{\bar{u} \tau_{s}}\right)\right]
\end{aligned}
$$

If this result is substituted into Eqn. (42) and the integrations with respect to $\omega_{1}, \omega_{2}, t_{1}$ and 
$t_{2}$ are performed, we obtain,

$$
\begin{aligned}
S(\mathbf{x}, \omega)= & -\frac{2 \pi \sqrt{\pi}}{\rho_{o} c_{o}} \iiint \iiint p_{a}\left(\mathbf{x}_{1}, \mathbf{x},-\omega\right) p_{a}\left(\mathbf{x}_{2}, \mathbf{x}, \omega\right) \frac{\hat{q}_{s}^{2}}{c^{2}} \ell_{s} \bar{u} \\
& \times \exp \left(-\frac{\omega^{2} \ell_{s}^{2}}{4 \bar{u}^{2}}\right) \exp \left[-\frac{1}{\ell_{s}^{2}}\left(\eta^{2}+\zeta^{2}\right)\right] \\
& \times \frac{\partial^{2}}{\partial \xi^{2}}\left[\exp \left(-\frac{|\xi|}{\bar{u} \tau_{s}}\right)\right] \exp \left(-i \frac{\omega \xi}{\bar{u}}\right) d \mathbf{x}_{1} d \mathbf{x}_{2}
\end{aligned}
$$

The adjoint solutions may be replaced by the form given by Eqn. (50). Then the integrations with respect to $\mathbf{x}_{1}$ may be replaced by integrations with respect to $\boldsymbol{\xi}$. The integrations with respect to $\eta$ and $\zeta$ are readily performed as before. However, the integration with respect to $\xi$ now takes the form,

$$
I=\int_{-\infty}^{\infty} \frac{\partial^{2}}{\partial \xi^{2}}\left[\exp \left(-\frac{|\xi|}{\bar{u} \tau_{s}}\right)\right] \exp \left(-i \frac{\omega \xi}{\bar{u}}\right) d \xi
$$

This integral may be rewritten in terms of a new variable $z=\xi /\left(\bar{u} \tau_{s}\right)$, such that,

$$
I=\frac{1}{\bar{u} \tau_{s}} \int_{-\infty}^{\infty} \frac{\partial^{2}}{\partial z^{2}}[\exp (-|z|)] \exp \left(-i \omega \tau_{s} z\right) d z
$$

Since the first term in the integrand must be treated as a generalized function, due to its discontinuous behavior at $\xi=0$, integration by parts should be performed before the integral may be evaluated. This is discussed in more detail in an Appendix to this paper. This gives,

$$
\begin{aligned}
I & =\frac{1}{\bar{u} \tau_{s}} \int_{-\infty}^{\infty} \exp (-|z|) \frac{\partial^{2}}{\partial z^{2}}\left[\exp \left(-i \omega \tau_{s} z\right)\right] d z \\
& =-\frac{\omega^{2} \tau_{s}}{\bar{u}} \int_{-\infty}^{\infty} \exp \left(-|z|-i \omega \tau_{s} z\right) d z
\end{aligned}
$$

The integral may now be evaluated by separating the integral into the contribution from 
$-\infty<\xi<0$ and that from $0<\xi<\infty$. This yields,

$$
I=-\frac{2 \omega^{2} \tau_{s}}{\bar{u}\left(1+\omega^{2} \tau_{s}^{2}\right)}
$$

The use of Eqn. (50) for the magnitude of the adjoint pressure then gives the final result for the spectral density,

$$
S(\mathbf{x}, \omega)=\frac{\sqrt{\pi}}{16 \pi^{2}} \frac{1}{\rho_{o} c_{o}^{5} x^{2}} \iiint \frac{\hat{q}_{s}^{2} \ell_{s}^{3}}{c^{2} \tau_{s}^{3}} \frac{\left(\omega \tau_{s}\right)^{4}}{\left(1+\omega^{2} \tau_{s}^{2}\right)} \exp \left(-\frac{\omega^{2} \ell_{s}^{2}}{4 \bar{u}^{2}}\right) d \mathbf{x}_{2}
$$

If $\hat{q}_{s}^{2} / c^{2}$ is set equal to $A^{2} \rho_{s}^{2} u_{s}^{4}$ this is exactly the result given by Eqn. (68) for Model II based on the acoustic analogy described in the previous section.

Thus it may be stated that: both Model II based on the acoustic analogy and Tam and Auriault's model ${ }^{1}$ yield identical noise prediction formulas at 90 degrees to the jet axis if consistent assumptions are made in the statistical description of the turbulent sources.

\section{Discussion}

In the previous section it was shown that both a model based on an acoustic analogy and Tam and Auriault's model ${ }^{1}$ yield identical noise prediction formulas if consistent assumptions are made concerning the statistical description of the turbulent noise sources. This might appear to be a somewhat negative result, as it shows that the recent model by Tam and Auriault ${ }^{1}$ would give as poor a prediction as models based on the acoustic analogy for the same source description. However, it could be viewed as providing guidance on what assumptions should be made in the source description if good predictions are to be made. One could obtain a noise prediction formula identical to that given by Tam and Auriault ${ }^{1}$ in Eqn. (52) in a number of ways. For example, only two of the spatial derivatives acting on the Lighthill source term correlation in Eqn. (59) need to be transferred to the Green's functions. Then an assumption could be made that the two-point cross correlation of the new "source" term 
is given by

$$
\left\langle\frac{\partial T_{i j}}{\partial x_{i}}\left(\mathbf{x}_{1}, \mathbf{t}_{1}\right) \frac{\partial T_{k \ell}}{\partial x_{k}}\left(\mathbf{x}_{2}, \mathbf{t}_{2}\right)\right\rangle=A_{j k} \frac{\rho_{s}^{2} u_{s}^{4}}{\ell_{s}^{2}} \exp \left\{-\frac{|\xi|}{\bar{u} \tau_{s}}-\frac{1}{\ell_{s}^{2}}\left[(\xi-\bar{u} \tau)^{2}+\eta^{2}+\zeta^{2}\right]\right\}
$$

However, this is just as arbitrary an assumption as that made by Tam and Auriault ${ }^{1}$ in proposing the form of two-point cross correlation given by Eqn. (43). Alternatively, we need to find a form of two-point cross correlation function for the Lighthill stress tensor that would give the form assumed by Tam and Auriault ${ }^{1}$ when the convective derivative operations are applied, as in Eqn. (72). This is no easy matter because of the discontinuous derivative of the assumed correlation at $\xi=0$. However, as noted by one reviewer, $|\xi|$ could be replaced by $\sqrt{\xi^{2}+\delta^{2}}$ and letting $\delta \rightarrow 0$ in the final result.

In the remainder of this final section we propose an acoustic analogy formulation that includes, as a part, the source description proposed by Tam and Auriault ${ }^{1}$ : but, it also identifies other physical mechanisms that are equally good candidates for noise sources. Other formulations of this type could be proposed: however, our formulation is based on equations of motion written in terms of the logarithm of the pressure.

The equations of continuity, momentum and energy, and the equation of state for a perfect gas, may be rearranged in the form,

$$
\frac{D \pi}{D t}=-\frac{\partial u_{i}}{\partial x_{i}}
$$

and

$$
\frac{D u_{i}}{D t}=-c^{2} \frac{\partial \pi}{\partial x_{i}}
$$

where

$$
\pi=\frac{1}{\gamma} \ln \left(\frac{p}{p_{o}}\right),
$$




$$
\frac{D}{D t} \equiv \frac{\partial}{\partial t}+u_{j} \frac{\partial}{\partial x_{j}}
$$

and $p_{o}$ is the mean static pressure that is assumed to constant. Equations (84) and (85) may be recast in the form of a third-order equation [Lilley, ${ }^{9}$ Colonius et al. ${ }^{15}$,

$$
\frac{D}{D t}\left[\frac{D^{2} \pi}{D t^{2}}-\frac{\partial}{\partial x_{i}}\left(c^{2} \frac{\partial \pi}{\partial x_{i}}\right)\right]-2 \frac{\partial u_{i}}{\partial x_{j}}\left[\frac{\partial}{\partial x_{j}}\left(c^{2} \frac{\partial \pi}{\partial x_{i}}\right)\right]=2 \frac{\partial u_{j}}{\partial x_{i}} \frac{\partial u_{k}}{\partial x_{j}} \frac{\partial u_{i}}{\partial x_{k}}
$$

If the variables in this equation are linearized about the mean thermodynamic properties and a parallel mean flow, given by,

$$
u(\mathbf{x}, t)=U\left(x_{2}, x_{3}\right) \delta_{i 1}+u_{i}^{\prime}(\mathbf{x}, t)
$$

Lilley's equation $^{9}$ may be obtained in the form

$$
\frac{D_{o}}{D t}\left[\frac{D_{o}^{2} \pi^{\prime}}{D t^{2}}-\frac{\partial}{\partial x_{i}}\left(\overline{c^{2}} \frac{\partial \pi^{\prime}}{\partial x_{i}}\right)\right]+2 \overline{c^{2}}\left\{\frac{d U}{d x_{2}} \frac{\partial^{2} \pi^{\prime}}{\partial x_{1} \partial x_{2}}+\frac{d U}{d x_{3}} \frac{\partial^{2} \pi^{\prime}}{\partial x_{1} \partial x_{3}}\right\}=\Gamma
$$

A detailed expansion of $\Gamma$ is given by Colonius et al. ${ }^{15}$ All terms in $\Gamma$ are at least second order in the fluctuations. In the limit of infinitesimal disturbances the equation reduces to a homogeneous equation that describes the propagation of sound in a parallel shear flow, such as that developed by Pridmore-Brown. ${ }^{16}$ Also, as noted by Colonius et al. ${ }^{15}$ it is in the form of an acoustic analogy since it is equivalent to the equation that describes the pressure fluctuations generated by an external distribution of stresses (and other source terms that are often neglected) imposed on a parallel shear flow.

Alternatively an acoustic analogy could be equally well formed by simply splitting the variables in Eqns. (84) and (85) into their mean and fluctuating components, assuming a parallel mean flow, and retaining only linear terms on the left hand side of the equation. 
This yields,

$$
\frac{D_{o} \pi^{\prime}}{D t}+\frac{\partial u_{i}^{\prime}}{\partial x_{i}}=-w^{\prime \prime}
$$

and

$$
\frac{D_{o} u_{i}^{\prime}}{D t}+\left(\frac{d U}{d x_{2}} u_{2}^{\prime}+\frac{d U}{d x_{3}} u_{3}^{\prime}\right) \delta_{i 1}+\overline{c^{2}} \frac{\partial \pi^{\prime}}{\partial x_{i}}=-f_{i}^{\prime \prime}
$$

with

$$
\frac{D_{o}}{D t} \equiv \frac{\partial}{\partial t}+U \frac{\partial}{\partial x_{1}}
$$

and

$$
w^{\prime \prime}=u_{j}^{\prime} \frac{\partial \pi^{\prime}}{\partial x_{j}} \quad \text { and } \quad f_{i}^{\prime \prime}=u_{j}^{\prime} \frac{\partial u_{i}^{\prime}}{\partial x_{j}}+c^{2^{\prime}} \frac{\partial \pi^{\prime}}{\partial x_{i}}
$$

The double prime notation is used to denote terms that are second order in the fluctuations. The term $w^{\prime \prime}$ represents the net rate of work done at second order by the fluctuating pressure on a fluid element. The term $f_{i}^{\prime \prime}$ is the same as that obtained by Goldstein, ${ }^{17}$ being equivalent to an externally applied force per unit mass acting on a parallel sheared mean flow. This term may also be written,

$$
f_{i}^{\prime \prime}=-\varepsilon_{i j k} u_{j}^{\prime} \omega_{k}^{\prime}+\frac{\partial}{\partial x_{i}}\left(\frac{u_{j}^{\prime} u_{j}^{\prime}}{2}\right)+c^{2^{\prime}} \frac{\partial \pi^{\prime}}{\partial x_{i}}
$$

where $\varepsilon_{i j k}$ is the alternating tensor and $\omega_{k}^{\prime}$ is the vorticity tensor. The first term in the total fluctuating force (per unit mass) is called the "vortex force" (also written in vector notation as $\left.\mathbf{u}^{\prime} \times \boldsymbol{\omega}^{\prime}\right)$. It is associated with the transport of rotating fluid elements by the fluctuating velocity field. The second term is the force generated by the second order dynamic head. It should be noted that this term is essentially the same single source term proposed by Tam and 
Auriault. ${ }^{1}$ The last term in Eqn. (95) is a source associated with temperature fluctuations in the turbulent flow as discussed by Tester and Morfey. ${ }^{18}$ Note that $c^{2^{\prime}}=\gamma R T^{\prime}$. This source term is an important component of the semi-empirical noise prediction model developed by Morfey et al. ${ }^{19}$ This last term appears as a source in the framework of an acoustic analogy. However, it is readily identified as the effect of sound propagation through a medium with a fluctuating speed of sound.

Equations (91) and (92) could be rearranged into a single inhomogeneous equation for $\pi^{\prime}$, exactly in the form of Eqn. (90). However, in their unmanipulated form it is easier to see the equivalent sources in the momentum and energy equations. The source term $\Gamma$, given by Colonius et al., ${ }^{15}$ is reproduced exactly by this rearrangement ${ }^{1}$. (To obtain the form given by Colonius et al. ${ }^{15}$ the source associated with speed of sound fluctuations is neglected as are third and higher order terms.) The complexity of $\Gamma$ is formidable. In its simplified form,

$$
\Gamma \approx \frac{D_{o}}{D t}\left(\frac{\partial u_{i}^{\prime} u_{j}^{\prime}}{\partial x_{i} \partial x_{j}}\right)-2\left(\frac{d U}{d x_{2}} \frac{\partial u_{2}^{\prime} u_{j}^{\prime}}{\partial x_{1} \partial x_{j}}+\frac{d U}{d x_{3}} \frac{\partial u_{3}^{\prime} u_{j}^{\prime}}{\partial x_{1} \partial x_{j}}\right)
$$

This has been interpreted as a quadrupole source distribution with components referred to as self- and shear-noise. However, the form of source term given by Eqn. (96) is simply the result of recasting the original inhomogeneous, linearized Euler equations given by Eqns. (91) and (92). In that primitive form there is no source term, or by inference, no source mechanism, associated with the mean velocity gradient, or any other mean flow property. All equivalent source terms are second order in the fluctuations only. In summary, the acoustic analogy formed by Eqns. (91) and (92) describes acoustic radiation from a sheared mean flow due to externally-applied forces and work performed on the fluid.

Since the source terms on the right hand sides of Eqns. (91) and (92) are not all in

\footnotetext{
${ }^{1}$ There appears to be a minor typographical error in the final source term component $\mathrm{Vb}$, that should be $\frac{\partial \pi^{\prime}}{\partial x_{1}}\left(u_{i}^{\prime} \frac{\partial u_{2}^{\prime}}{\partial x_{i}}\right)+u_{2}^{\prime} \frac{\partial}{\partial x_{1}}\left(u_{i}^{\prime} \frac{\partial \pi^{\prime}}{\partial x_{i}}\right)$
} 
the form of the divergence of a scalar, as in Tam and Auriault's model, ${ }^{1}$ it is not possible to follow the simplifying analysis of Eqns. (35) to (40). In addition, since there is more than one source term, their correlation must be considered when the autocorrelation of the far field pressure is formed. Previous analyses have considered each source mechanism to be statistically independent and that approach, though somewhat questionable, could be followed.

The scaling of the source terms, based on the CFD solution, may also be addressed. The first two components of $f_{i}^{\prime \prime}$ of Eqn. (95) should be scaled by

$$
k / \ell_{s}
$$

The last term should be scaled by

$$
\frac{\left(T_{s}-T_{o}\right)}{T_{o}} \frac{\rho_{s}}{\rho_{o}} \frac{k}{\ell_{s}}
$$

where, $T_{s}$ is the mean temperature in the source volume element. It should be noted that this is only distinguished from the scaling for the first source terms (or the source term in Tam and Auriault's model ${ }^{1}$ ) by the local relative temperature difference. Finally, the unsteady work source term in Eqn. (91) should scale with

$$
\frac{1}{c_{o}^{2}} \frac{\rho_{s}}{\rho_{o}} \frac{k^{3 / 2}}{\ell_{s}}
$$

There are several issues that remain to be addressed. Though the present paper has considered the noise radiation at $90^{\circ}$ to the jet axis, so that mean flow/acoustic interaction effects may be neglected, there are distinct differences between the traditional (Model I) formulation and Tam and Auriault's model ${ }^{1}$ at other angles to the jet axis. In the latter 
model, the variation of spectral density with polar angle is contained in the factor

$$
\frac{\left|p_{a}\left(x_{2}, x, \omega\right)\right|^{2}}{\left[1+\left(\omega^{2} / \omega_{s}^{2}\right)\left(1-\bar{u} \cos \theta / c_{o}\right)^{2}\right]}
$$

Away from the zone of silence there is little angular variation in $\left|p_{a}\left(x_{2}, x, \omega\right)\right|$ at a given frequency as shown by Tam and Auriault. ${ }^{14}$ The factor in the denominator in Eqn. (100) is a very weak function of polar angle. For example, with $\bar{u} / c_{o}=1.0$ there is only a 2 decibel change in the spectral density for $\omega / \omega_{s}=1.0$ and $\theta=60^{\circ}$ relative to the value at $90^{\circ}$. This is consistent with the experimental spectra shown by Tam and Auriault. ${ }^{14}$ However, the traditional models based on Lighthill's acoustic analogy suggest a directivity that varies as $\left(1-M_{c} \cos \theta\right)^{-5}$. If we let $M_{c}=0.62 U_{j} / c_{o}\left(\right.$ Goldstein $\left.^{7}\right)$ then a relative change of 8 decibels is predicted for the same conditions. This is much greater than the measured variation. Further analysis by Goldstein ${ }^{7}$ to include the mean flow effects changes the convection factor from $\left(1-M_{c} \cos \theta\right)^{-5}$ to $\left(1-M_{c} \cos \theta\right)^{-3}$. This results in predictions for the directivity of the overall intensity in better agreement with experiment. In addition, in traditional models based on the acoustic analogy, there is no separation of source mechanisms between contributions from small-scale or large-scale turbulence. So predictions are made at all polar angles for the same assumed source. Whereas, in Tam and Auriault's model, ${ }^{14}$ predictions are limited to angles approximately greater than $60^{\circ}$ to the jet downstream axis. Closer to the jet axis Tam and Auriault argue that a noise mechanism associated with the large-scale turbulence is present. It should be noted that Model II based on the acoustic analogy, described in Section 4, gives a convection factor identical to that of Tam and Auriault. The reason for the difference is tied to the choice of two-point cross correlation factor for the sources. In Model I the form of the cross correlation is assumed in a moving frame of reference. Whereas, in Model II, the form of the cross correlation is assumed in a reference frame fixed to the jet nozzle. The fact that the two models give different convection factors indicates that one or other of the assumed forms is incorrect or insufficiently accurate. 
The moving and fixed reference frame cross correlations are related simply by a coordinate transformation. So consistent choices are possible and the final result must be independent of the reference frame. It is readily shown that the usual Gaussian form of cross correlation in the moving frame gives a fixed frame correlation that is consistent with Eqn. (71) and measurements. Since, as noted above, the temporal variations of the sources that determine the sound radiation are more clearly identifiable in a moving reference frame, it appears to be the appropriate choice: and with its selection comes convective amplification. Finally it should be noted that the measured two-point correlations should contain contributions from both the large-scale and small-scale turbulence. The wavenumber/frequency spectrum that provides the distinction between radiating and non-radiating components of the turbulent field [see Eqn. (17)] should be able to describe both components if it is chosen appropriately. The extraction of two-point correlations from either experimental or simulation data for high speed jets, where it known that the instability wave radiation is present, would be very illuminating. Thus, the issues of the appropriate convection factor and the separate contributions, if they are separate, of the small-scale and large-scale noise generation mechanisms remain open questions.

In this paper we have tried to reconcile the apparent differences between models based on the acoustic analogy and alternative models. We have shown that there is no difference in the eventual noise prediction formulas if consistent assumptions are made concerning the statistical properties of the turbulent noise sources. We have also proposed an acoustic analogy that provides a clear representation of equivalent source mechanisms. We have also tried to identify some of the remaining issues that have yet to be resolved and have proposed measurements or simulations that would help to resolve these issues. The recent reexamination of models based on the acoustic analogy, much of it prompted by the success of the model proposed by Tam and Auriault ${ }^{1}$ has been very valuable. The authors hope that this paper will stimulate further work that will result in an improved understanding of how to predict radiated noise using CFD simulations. 


\section{Appendix. The Use of Generalized Differentiation in the Algebraic Manipulation of Equation (78)}

Consider the following integral depending on the variable $y$ and a real variable $\beta$

$$
\begin{aligned}
f(y) & =\int_{-\infty}^{\infty} \exp (-|x-y|+i \beta x) d x \\
& =\frac{2 \exp (i \beta y)}{1+\beta^{2}}
\end{aligned}
$$

It is clear that the second derivative of the function on the left hand side of Eqn. (102) is

$$
\frac{d^{2} f}{d y^{2}}(y)=-\frac{2 \beta^{2} \exp (i \beta y)}{1+\beta^{2}}
$$

If we differentiate Eqn. (101) with respect to the variable $y$ we obtain

$$
\int_{-\infty}^{\infty} \frac{d^{2}}{d y^{2}}\{\exp (-|x-y|+i \beta x)\} d x
$$

It might appear to be possible to split this integral into two parts giving,

$$
\int_{-\infty}^{y} \frac{d^{2}}{d y^{2}}\{\exp (x-y+i \beta x)\} d x+\int_{y}^{\infty} \frac{d^{2}}{d y^{2}}\{\exp (-x+y+i \beta x)\} d x=\frac{2 \exp (i \beta y)}{1+\beta^{2}}
$$

This does not agree with the result given by Eqn. (103). To determine why this is we note that the second derivative of the function $\exp (-|x-y|)$ is discontinuous at $x=y$ and thus taking the second derivative inside the integral is not valid. We should have kept the derivatives outside the integral, split the range of integration, and then used the Leibniz rule 
of differentiation under the integral sign twice to get,

$$
\begin{aligned}
& \frac{d^{2}}{d y^{2}} \int_{-\infty}^{\infty} \exp (-|x-y|+i \beta x) d x \\
= & \frac{d^{2}}{d y^{2}}\left\{\int_{-\infty}^{y} \exp (x-y+i \beta x) d x+\int_{y}^{\infty} \exp (-x+y+i \beta x) d x\right\} \\
= & -\frac{2 \beta^{2} \exp (i \beta y)}{1+\beta^{2}}
\end{aligned}
$$

We have obtained the correct result but only at the expense of more algebraic manipulations. We want to be able to obtain the correct result when we change the order of differentiation and integration without the added burden of book-keeping when our integrand is discontinuous. This can be achieved as follows.

It is seen that $f(y)$ is an analytic function. This means that ordinary and generalized derivatives of this function are identical. However, we can exchange the order of integration and generalized differentiation. Using a bar over the differentiation sign to denote generalized differentiation, we have now the following mathematically correct manipulation:

$$
\begin{aligned}
\frac{d^{2} f}{d y^{2}}(y) & =\int_{-\infty}^{\infty} \frac{\bar{d}^{2}}{d y^{2}}\{\exp (-|x-y|+i \beta x)\} d x \\
& =\int_{-\infty}^{\infty} \frac{\bar{d}^{2}}{d x^{2}}\{\exp -|x-y|\} e^{i \beta x} d x \\
& =\int_{-\infty}^{\infty} \exp -|x-y| \frac{\bar{d}^{2}}{d x^{2}}\left\{e^{i \beta x}\right\} d x \\
& =-\frac{2 \beta^{2} \exp (i \beta y)}{1+\beta^{2}}
\end{aligned}
$$

So we have obtained the same result as Eqn. (103).

In this paper we have obtained an equation similar to Eqn. (104) and then we claimed that the second derivative with respect to the variable $y$ must be treated as a generalized 
derivative. The purpose of this appendix is to justify this claim. The root of the problem in Eqn. (104) is that the interchange of the order of limit operations of integration and ordinary differentiation is not permissible because of the discontinuity of the second derivative of the integrand at $x=y$. However, in this paper, we have freely interchanged the order of integration and ordinary differentiation without any attempt at justification. For example, consider the derivation of the relation,

$$
\begin{aligned}
\left\langle\frac{\partial^{2} T_{x x}}{\partial t^{2}}\left(\mathbf{y}_{1}, t_{1}\right) \frac{\partial^{2} T_{x x}}{\partial t^{2}}\left(\mathbf{y}_{2}, t_{2}\right)\right\rangle & =\frac{\partial^{4}}{\partial \tau^{4}}\left\langle T_{x x}\left(\mathbf{y}_{1}, t\right) T_{x x}\left(\mathbf{y}_{2}, \tau_{o}\right)\right\rangle \\
\tau_{o} & =t+\tau+\frac{\mathbf{x}}{x} \cdot \frac{\left(\mathbf{y}_{2}-\mathbf{y}_{1}\right)}{c_{o}}
\end{aligned}
$$

This is necessary in proceeding from Eqn. (6) to Eqn. (9) in the paper. We have performed two integrations by parts with respect to $t$, replaced the fourth order differentiation with respect to $t$ with the same order differentiation with respect to $\tau$, and then we exchanged the order of differentiation with the integration for time averaging (see Goldstein, ${ }^{7}$ Appendix 2). These operations can only be justified for integrands whose derivatives are continuous. More precisely, the uniform convergence of the integrals must be considered. As has happened in this paper, we may next introduce an algebraic expression for the statistical properties of the turbulence and perform algebraic manipulations that involve bringing into integrals derivatives of some order of a variable. We can do this easily and without the burden of the detailed analysis of classical calculus, exhibited in the manipulations of Eqn. (106), if we treat all derivatives from the start as generalized derivatives. Practically all the analysis remains the same except in situations like the exchange of the order of limit operations where we must indicate generalized differentiation explicitly as we have done here. The situation is completely analogous to linear aerodynamic theory where occasionally we have to interpret divergent integrals as the finite part of divergent integrals. The reason is that divergent integrals appear if improper exchange of the order of differentiation and integration is performed. The finite part procedure gives the same result as if the derivative was kept 
outside the integral. The procedure itself is obtained by assuming that the derivative is a generalized derivative and can, therefore, be taken inside the integral and interpreted by the rules of generalized function theory.

Readers should consult Canonical Regularization in Gelfand and Shilov, ${ }^{20}$ for the theoretical background relevant to the subject of this appendix. 


\section{References}

[1] Tam, C. K. W. and Auriault, L., "Jet Mixing Noise From Fine-Scale Turbulence," AIAA Journal, Vol. 37, No. 2, 1999, pp. 145-153.

[2] Lighthill, M. J., "On Sound Generated Aerodynamically: I. General Theory," Proc. Roy. Soc. Lond. A, Vol. 211, 1952, pp. 564-587.

[3] Lighthill, M. J., "On Sound Generated Aerodynamically. II. Turbulence as a Source of Sound," Proc. Roy. Soc. Lond. A, Vol. 222, 1954, pp. 1-32.

[4] Balsa, T. F. and Gliebe, P. R., "Aerodynamics and Noise of Coaxial Jets," AIAA Journal, Vol. 15, No. 11, 1977, pp. 1550-1558.

[5] Khavaran, A., Krejsa, E. A., and Kim, C. M., "Computation of Supersonic Jet Mixing Noise for an Axisymmetric Convergent Divergent Nozzle," J. Aircraft, Vol. 31, No. 3, 1994, pp. 603.

[6] Khavaran, A., "Role of Anisotropy in Turbulent Mixing Noise," AIAA Journal, Vol. 37, No. 7, 1999, pp. 832-841.

[7] Goldstein, M. E., Aeroacoustics, McGraw-Hill, New York, 1976.

[8] Hubbard, H. H., Aeroacoustics of Flight Vehicles: Volume 1: Noise Sources, Acoustical Society of America, Woodbury, NY, 1995.

[9] Lilley, G. M., "On the Noise From Jets," Noise Mechanisms, AGARD-CP-131, Mar. 1974, pp. 13.1-13.12.

[10] Ffowcs Williams, J. E., "The Noise From Turbulence Convected at High Speed," Phil. Trans. A, Vol. 255, 1963, pp. 469-503. 
[11] Tanna, H. K., Dean, P. D., and Burrin, R. H., "The Generation and Radiation of Supersonic Jet Noise. Vol. III, Turbulent Mixing Noise Data," 1976, AFAPL-TR-76-65Vol III.

[12] Thies, A. T. and Tam, C. K. W., "Computation of turbulent axisymmetric and nonaxisymmetric jet flows using the $k-\epsilon$ model," AIAA Journal, Vol. 34, 1996, pp. 309-316.

[13] Tam, C. K. W., Golebiowski, M., and Seiner, J. M., "On the Two Components of Turbulent Mixing Noise from Supersonic Jets," AIAA Paper 96-1716, 1996.

[14] Tam, C. K. W. and Auriault, L., "Mean Flow Refraction Effects on Sound Radiated From Localized Sources in a Jet," J. Fluid Mech., Vol. 370, 1998, pp. 149-174.

[15] Colonius, T., Lele, S. K., and Moin, P., "Sound Generation in a Mixing Layer," J. Fluid Mech., Vol. 330, 1997, pp. 375-409.

[16] Pridmore-Brown, D. C., "Sound Propagation in a Fluid Flowing Through an Attenuating Duct," J. Fluid Mech., Vol. 4, 1958, pp. 393-406.

[17] Goldstein, M. E., "Aeroacoustics of Turbulent Shear Flows," Annual Review of Fluid Mechanics, Vol. 16, 1984, pp. 263-285.

[18] Tester, B. J. and Morfey, C. L., "Developments in Jet Noise Modeling - Theoretical Predictions and Comparisons With Measured Data," J. Sound and Vibration, Vol. 46, No. 1, 1976, pp. 79-103.

[19] Morfey, C. L., Szewczyk, V. M., and Tester, B. J., "New Scaling Laws for Hot and Cold Jet Mixing Noise Based on a Geometrics Acosutics Model," J. Sound Vib., Vol. 61, No. 2, 1978, pp. 255-292.

[20] Gel'fand, I. M. and Shilov, G. E., Generalized Functions: Properties and Operations, Vol. 1, Academic Press, New York, 1964. 


\section{List of Figures}

1 Comparison With Experimental Data of the Predicted Spectral Density at $90^{\circ}$ to the Jet Axis Using Model I Based on the Acoustic Analogy. $x=72 D_{j}$, $M_{j}=0.911, T_{j} / T_{o}=0.975, D_{j}=0.0508 \mathrm{~m} . \bigcirc-\bigcirc$, Prediction; $\bullet$, Experimental, Tanna et al. ${ }^{11} \ldots \ldots \ldots$. . . . . . . . . . . . . 39

2 Prediction of Spectral Density at $90^{\circ}$ to the Jet Axis Using Model I Based on the Acoustic Analogy Showing the Contribution to the Spectrum in One Diameter Slices. The Contributions From Slices for the First Twenty Diameters are Shown in Alternating Solid and Dashed Lines. $x=72 D_{j}, M_{j}=0.911$, $T_{j} / T_{o}=0.975, D_{j}=0.0508 \mathrm{~m}$. O, Overall Prediction. .......

3 Comparison With Experimental Data of the Predicted Spectral Density at $90^{\circ}$ to the Jet Axis Using Tam and Auriault's Model. ${ }^{1} x=72 D_{j}, M_{j}=$ $0.911, T_{j} / T_{o}=0.975, D_{j}=0.0508 \mathrm{~m} . \square \square \square$, Present prediction using coefficients determined by comparison with Tanna et al. ${ }^{11}$ data; $\diamond-$ $\diamond$, Prediction based on coefficients determined by Tam and Auriault; ${ }^{1} \bullet$, Experiment, Tanna et al. ${ }^{11} \ldots \ldots \ldots$. . . . . . . . . . . . . 41

4 Comparison With Experimental Data of the Predicted Spectral Density at $90^{\circ}$ to the Jet Axis Using Models I and II Based on the Acoustic Analogy and Tam and Auriault's Model. ${ }^{1} x=72 D_{j}, M_{j}=0.911, T_{j} / T_{o}=0.975$, $D_{j}=0.0508 \mathrm{~m} . \bigcirc \quad \bigcirc$, Model I; $\triangle \longrightarrow \triangle$, Model II; $\square \longrightarrow \square$, Tam and Auriault's Model; •, Experiment, Tanna et al. ${ }^{11} \ldots$. . . . . . . . . . . 42 


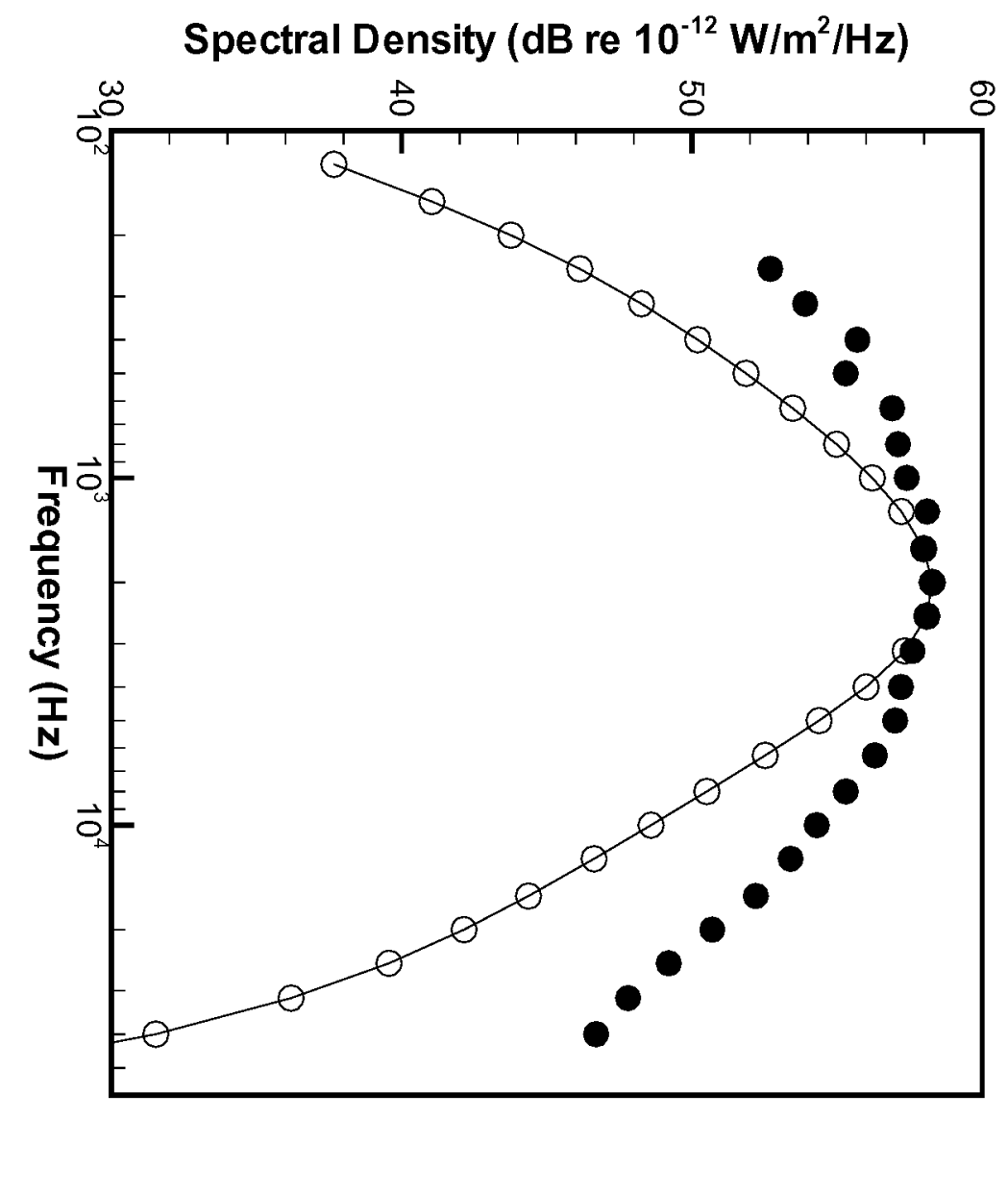




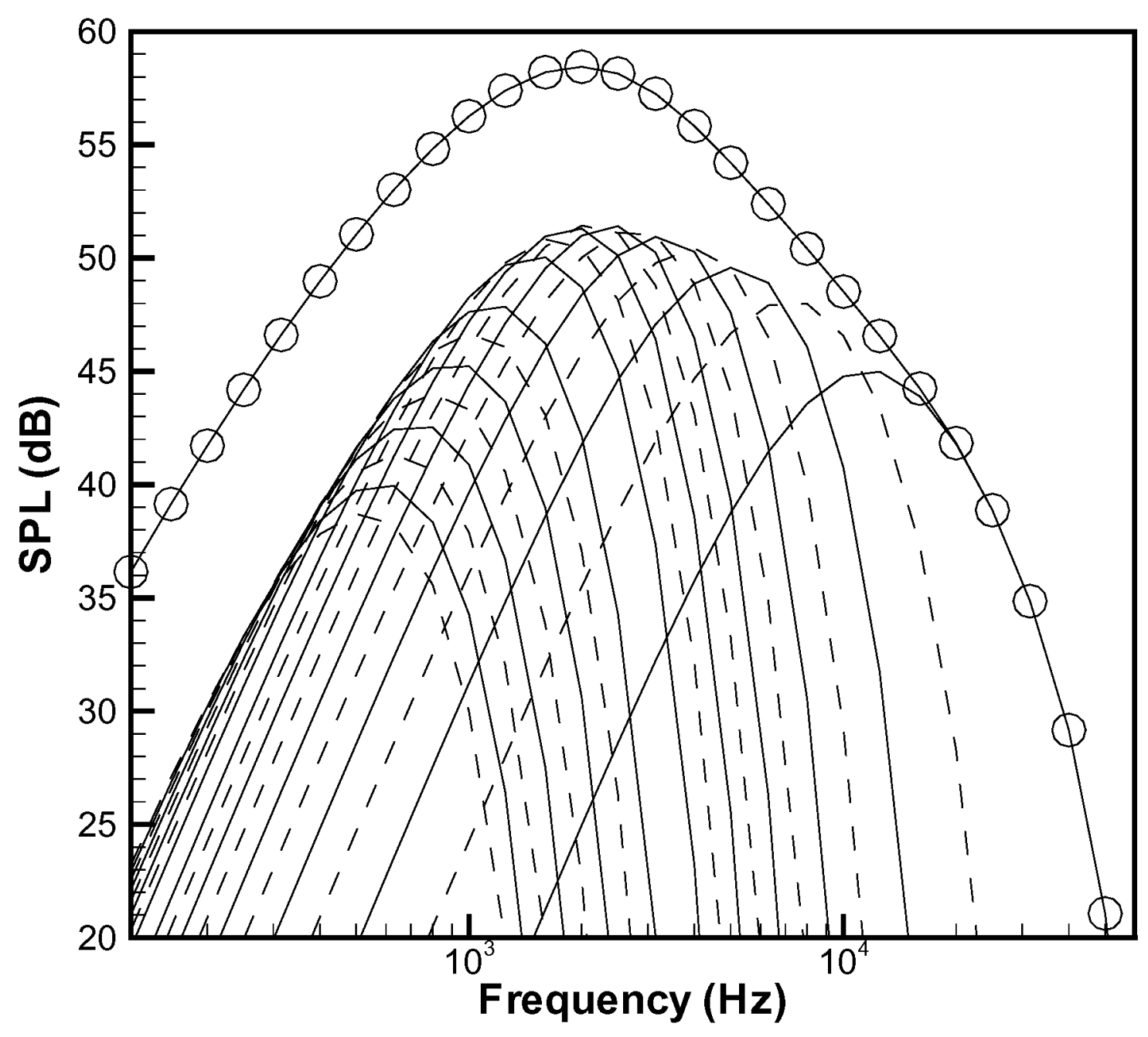

Figure 2: Prediction of Spectral Density at $90^{\circ}$ to the Jet Axis Using Model I Based on the Acoustic Analogy Showing the Contribution to the Spectrum in One Diameter Slices. The Contributions From Slices for the First Twenty Diameters are Shown in Alternating Solid and Dashed Lines. $x=72 D_{j}, M_{j}=0.911, T_{j} / T_{o}=0.975, D_{j}=0.0508 \mathrm{~m} . \bigcirc \quad \bigcirc$, Overall Prediction. 


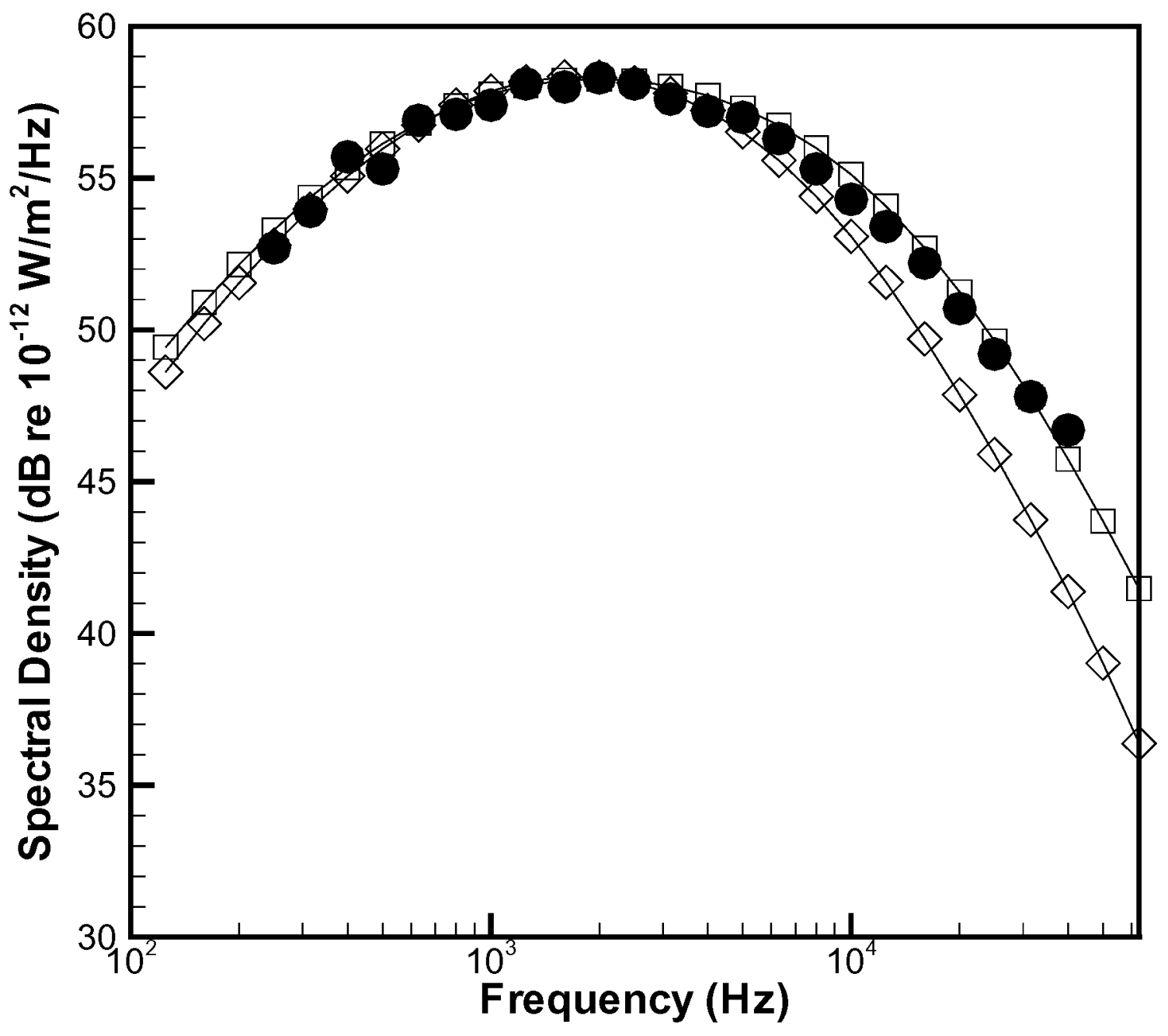

Figure 3: Comparison With Experimental Data of the Predicted Spectral Density at $90^{\circ}$ to the Jet Axis Using Tam and Auriault's Model. ${ }^{1} x=72 D_{j}, M_{j}=0.911, T_{j} / T_{o}=0.975$, $D_{j}=0.0508 \mathrm{~m} . \square-\square$, Present prediction using coefficients determined by comparison with Tanna et al. ${ }^{11}$ data; $\diamond — \diamond$, Prediction based on coefficients determined by Tam and Auriault; ${ }^{1} \bullet$, Experiment, Tanna et al. ${ }^{11}$ 


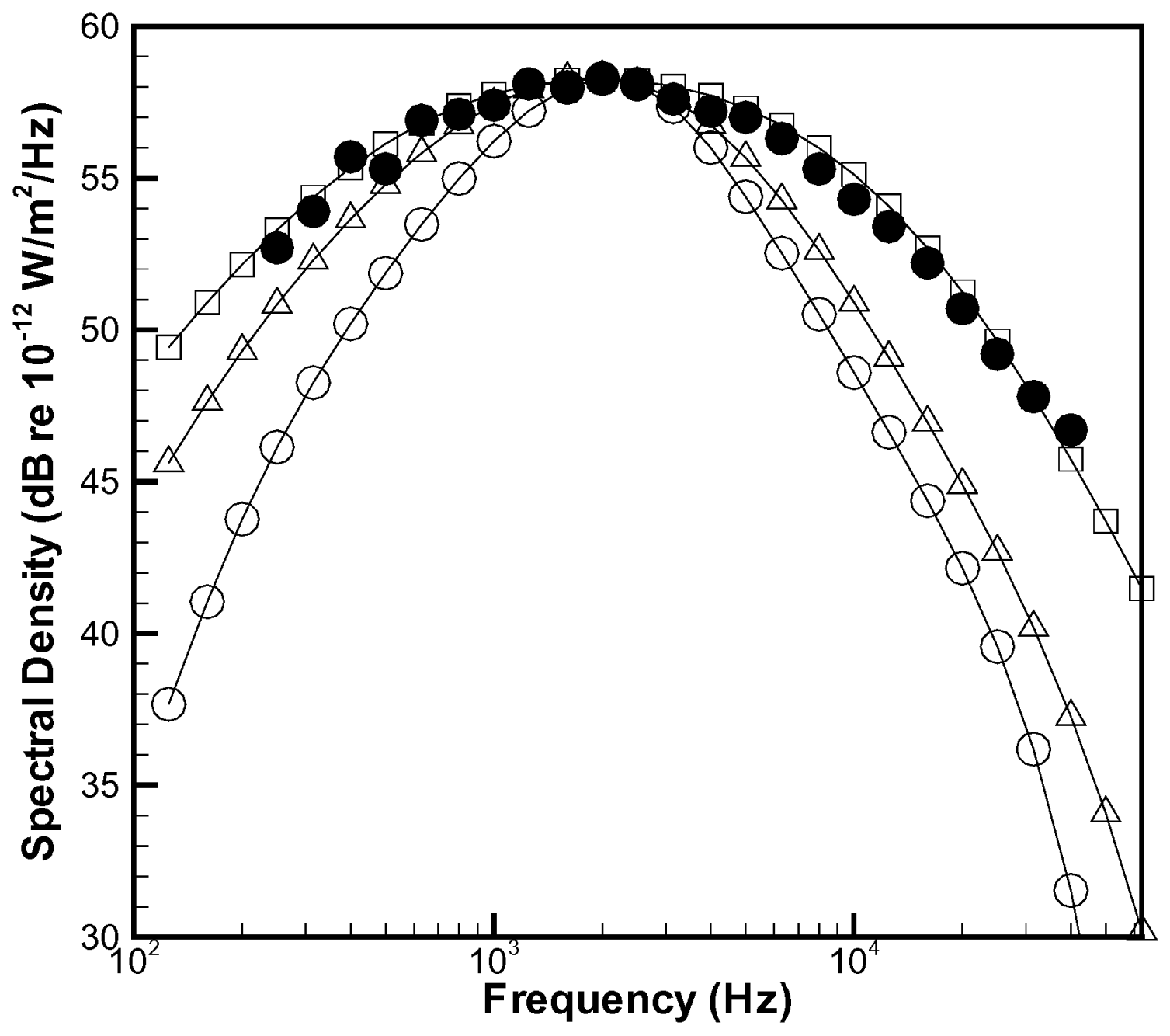

Figure 4: Comparison With Experimental Data of the Predicted Spectral Density at $90^{\circ}$ to the Jet Axis Using Models I and II Based on the Acoustic Analogy and Tam and Auriault's Model. $^{1} \quad x=72 D_{j}, M_{j}=0.911, T_{j} / T_{o}=0.975, D_{j}=0.0508 \mathrm{~m} . \bigcirc-\bigcirc$, Model I; $\triangle \backsim \triangle$, Model II; $\square \longrightarrow \square$, Tam and Auriault's Model; $\bullet$, Experiment, Tanna et al. 